\title{
CONSTRUCTION OF SELF-ADJOINT BEREZIN-TOEPLITZ OPERATORS ON KÄHLER MANIFOLDS AND A PROBABILISTIC REPRESENTATION OF THE ASSOCIATED SEMIGROUPS
}

\author{
BERNHARD G. BODMANN
}

\begin{abstract}
We investigate a class of operators resulting from a quantization scheme attributed to Berezin. These so-called Berezin-Toeplitz operators are defined on a Hilbert space of square-integrable holomorphic sections in a line bundle over the classical phase space. As a first goal we develop self-adjointness criteria for Berezin-Toeplitz operators defined via quadratic forms. Then, following a concept of Daubechies and Klauder, the semigroups generated by these operators may under certain conditions be represented in the form of Wiener-regularized path integrals. More explicitly, the integration is taken over Brownian-motion paths in phase space in the ultra-diffusive limit. All results are the consequence of a relation between Berezin-Toeplitz operators and Schrödinger operators defined by certain quadratic forms. The probabilistic representation is derived in conjunction with a version of the Feynman-Kac formula.
\end{abstract}

\section{IntRoduCtion}

1.1. Scope of this work. The general theme in this work is the geometric formulation of Berezin-Toeplitz quantization on Kähler manifolds. This quantization prescription was introduced by Berezin [1,2] to construct quantum models with the help of certain continuous representations in the sense of Klauder [3 [8], more specifically by using spaces of holomorphic functions on phase-space manifolds with a Kähler structure. Cahen, Gutt, Rawnsley and others [9 13] subsequently cast Berezin's construction in a manifestly coordinate-independent form by borrowing ideas from geometric quantization 14 [16]. In this form the quantum kinematics is encoded in a Hilbert space of square-integrable, holomorphic sections in a holomorphic line bundle. A Berezin-Toeplitz operator $T_{f}$ on such a Hilbert space is characterized by its associated sesquilinear form, which is obtained by multiplying the measure in the $L^{2}$-inner product of the Hilbert space with a sufficiently regular real-valued function $f$. The quantization context arises from interpreting this function as a classical observable that is in some sense in correspondence with $T_{f}$. Indeed, one may prove that the precise notion of a correspondence principle applies in the case of homogeneous or compact Kähler manifolds, see [2, 17] or [9, 18.

A first goal in this work is to derive conditions for the validity of this quantization procedure. More precisely, we obtain regularity conditions for possibly unbounded

Date: 31st October 2018.

Key words and phrases. Berezin-Toeplitz quantization, self-adjointness, Wiener-regularized path integrals; 81S10, 58D30 (MSC 2000). 
classical Hamiltonians ensuring that their quantum analogues are self-adjoint operators. The discussion of these conditions develops from a rather abstract level to concrete criteria in terms of the Kato class that is intrinsically determined by the underlying geometry.

The remaining part of this work generalizes an approach to path-integral quantization proposed by Daubechies and Klauder 192 23]; see also [24. Superficially, it is a phase-space version of Feynman's path integral that has been rendered mathematically well-defined by a Wiener-measure regularization. However, a closer look shows that the construction by Daubechies and Klauder can be understood as a path-integral formulation of Berezin-Toeplitz quantization on certain homogeneous Kähler manifolds. Indeed, a generalization to arbitrary Kähler manifolds has been advocated in several publications [25 28] and carried out for the compact case by Charles [29]. The advocated generalization is a probabilistic expression for the unitary group $\left\{e^{-i t T_{f}}\right\}_{t \in \mathbb{R}}$ generated by a Berezin-Toeplitz operator $T_{f}$. More precisely, a Wiener-regularized path integral expresses the integral kernel of the time-evolution operator $e^{-i t T_{f}}$ as the ultra-diffusive limit of an expectation value over Brownian motion paths on the classical phase space.

In contrast to the setting considered by Charles [29], we include the case of unbounded Berezin-Toeplitz operators and non-compact manifolds, subject to certain technical conditions. Moreover, we show that instead of the Brownian motion governed by the original Kähler metric as in [29], the Wiener regularization may be realized using a conformally rescaled metric, at the cost of adjusting the path measure with a suitable Feynman-Kac functional. A minor difference with the original intent of Daubechies and Klauder and its advocated generalizations [25 28] is that instead of unitary groups, we focus on the probabilistic representation of semigroups $\left\{e^{-t T_{f}}\right\}_{t \geq 0}$ that are generated by self-adjoint, semibounded Berezin-Toeplitz operators. The expression for $e^{-t T_{f}}$ is entirely geometric in nature and opens up a wealth of analytic tools from the extensively studied background of Brownian motion. One may expect that this probabilistic representation assumes a role in the investigation of Berezin-Toeplitz operators similar to that of the Feynman-Kac formula in the analysis of Schrödinger operators.

1.2. Structure and Contents. In Section 2 we we show that a class of coherent states is essential to the understanding of Berezin-Toeplitz quantization. After defining Berezin-Toeplitz operators in terms of semibounded quadratic forms, we give an abstract condition for their self-adjointness. Section 3 establishes a relationship between Berezin-Toeplitz and Schrödinger operators, which makes standard techniques from the context of differential operators available to formulate more concrete conditions ensuring the self-adjointness of a Berezin-Toeplitz operator. The main topic of Section 1 is the probabilistic representation of semigroups generated by self-adjoint, semibounded Berezin-Toeplitz operators. This result is called the Daubechies-Klauder formula. It is derived from a version of the Feynman-Kac formula for Schrödinger operators on Riemannian manifolds. Finally, we summarize the results in Section 5 and conclude with an outlook on further developments.

\section{Berezin-Toeplitz Quantization from a Coherent-State Perspective}

This section explains the construction of self-adjoint operators according to a quantization scheme in the spirit of Berezin 11,2. In a geometric formulation of 
this scheme [9 13], the underlying Hilbert space contains square-integrable, holomorphic sections in a holomorphic line bundle $\mathcal{L}$ with a compatible connection $\nabla$ over the classical phase space $\mathcal{M}$. The correspondence between the geometry of the line bundle and the classical phase-space structure is implicit in the fundamental assumption that the symplectic form on $\mathcal{M}$ can be reconstructed as a multiple of the curvature associated with the connection.

The first part of this section describes how a family of coherent states arises naturally with Berezin-Toeplitz quantization. Conversely, it is possible to recover some of the additional structures that are imposed on the classical phase space from the presence of such coherent states. The details are explained in the following exposition.

Klauder's concept of a continuous representation [3 8] is based on the existence of a family of orthogonal projectors $\left\{\Pi_{x}\right\}_{x \in \mathcal{M}}$ onto one-dimensional subspaces of a separable complex Hilbert space $\mathcal{H}$, indexed by points in a topological manifold such that $x \mapsto \Pi_{x}$ is weakly continuous. If there is a measure $\mathfrak{m}$ on $\mathcal{M}$ such that the integral $\int_{\mathcal{M}} \Pi_{x} d \mathfrak{m}(x)=\mathrm{id}_{\mathcal{H}}$ provides a weakly convergent resolution of the identity mapping $\operatorname{id}_{\mathcal{H}}$, then we call each one-dimensional subspace $e(x):=\Pi_{x} \mathcal{H}$ a coherent state. Thus, one can think of the manifold $\mathcal{M}$ as being embedded in the projective Hilbert space $P \mathcal{H}$, the set of all one-dimensional subspaces of $\mathcal{H}$. By definition, the image of the embedding constitutes the family of coherent states. The identification of collinear vectors in $\mathcal{H}$ to describe a (pure) quantum state induces additional structures on $\mathcal{M}$.

Since $P \mathcal{H}$ is the base manifold of a bundle $P: \mathcal{H} \backslash\{0\} \rightarrow P \mathcal{H}$, where the projection $P$ maps any nonzero vector in $\mathcal{H}$ to the one-dimensional subspace it generates, the embedding of $\mathcal{M}$ pulls back the fibers $\pi^{-1}(\{x\}):=P^{-1}(\{e(x)\}), x \in$ $\mathcal{M}$. To make $\mathcal{M}$ the base manifold of a complex line bundle, the missing zero vector must be inserted in every fiber $\mathcal{L}_{x}:=\pi^{-1}(\{x\}) \cup\{0\}$ and thus a bundle is created with total space $\mathcal{L}=\bigcup_{x \in \mathcal{M}} \mathcal{L}_{x}$ and projection $\pi$. If we suppose that the linear hull of $\mathcal{L}$ is dense in $\mathcal{H}$, then the linear functional $\vartheta_{v}: \psi \mapsto(v, \psi)$ restricted to $\psi \in \mathcal{L}$ provides a representation of $v \in \mathcal{H}$ as a function on $\mathcal{L}$ that is complex linear in the fibers. If $\mathcal{M}$ is a differentiable manifold and the mapping $x \mapsto \Pi_{x}$ is in some sense smooth, then as subsets of $\mathcal{H}$, the fibers in the total space $\mathcal{L}$ inherit additional features. The scalar product $(\cdot, \cdot)$ serves simultaneously as a Hermitian metric on both the total space $\mathcal{L}$ and the tangent space $T \mathcal{L}$. The notion of horizontal transport passes from $\mathcal{H}$ to $\mathcal{L}$, which takes a smooth curve $\zeta: \mathbb{R} \rightarrow \mathcal{M}$ together with a starting point $\hat{\zeta}(0)$ in $\pi^{-1}(\zeta(0))$ and produces the lifted curve $\hat{\zeta}$ in $\mathcal{L}$ by moving in an infinitesimal time step $d t$ from $\hat{\zeta}(t), t \in \mathbb{R}$, to the orthogonal projection of $\hat{\zeta}(t)$ onto the space $e(\zeta(t+d t))$. In fact, this way the norm of a horizontally transported vector in the fiber is left invariant while its base point moves along the curve in $\mathcal{M}$. In other words, the connection on the bundle corresponding to the horizontal transport is compatible with the Hermitian structure.

Berezin-Toeplitz quantization realizes a class of such continuous representations in a setting that is familiar in algebraic geometry [30: If $\mathcal{L}$ is a holomorphic line bundle over a Kähler manifold, then the curvature of the line bundle is a closed two-form [31]. This two-form is up to an imaginary factor assumed to be equal to the symplectic form on $\mathcal{M}$. The Hilbert space chosen by Berezin-Toeplitz quantization is the space of holomorphic sections that are square-integrable with respect to Liouville's measure, the maximal exterior power of the curvature form. These 
requirements are needed to show a correspondence principle for compact $\mathcal{M}[9,18$. Unfortunately, they also restrict the universality of Berezin-Toeplitz quantization. Not all symplectic manifolds can be equipped with a compatible complex structure, and even less may be obtained as the base manifold of a holomorphic line bundle such that its curvature is a constant multiple of the original symplectic form 18. In order to provide a resolution of the identity $\operatorname{id}_{\mathcal{H}}$ according to $\int_{\mathcal{M}} \Pi_{x} d \mathfrak{m}(x)=\mathrm{id}_{\mathcal{H}}$, the measure $\mathfrak{m}$ is chosen as a locally rescaled version of the Liouville form, see [11]. More generally, Berezin-Toeplitz quantization maps the classical observable represented by a bounded real-valued function $f: \mathcal{M} \rightarrow \mathbb{R}$ to the self-adjoint operator obtained from $T_{f}:=\int_{\mathcal{M}} f(x) \Pi_{x} d \mathfrak{m}(x)$. In both cases, the integral converges in the strong sense. The quantization of dynamics is then realized with the unitary group $\left\{e^{-i t T_{f}}\right\}_{t \in \mathbb{R}}$ that results from choosing $f$ as the generator of classical time evolution.

\subsection{Hilbert Spaces of Square-Integrable, Holomorphic Sections.}

Definition 1. Let us assume that a complex line bundle $\mathcal{L} \stackrel{\pi}{\rightarrow} \mathcal{M}$ is equipped with a Hermitian metric $h=\left\{h_{x}\right\}_{x \in \mathcal{M}}$ on its fibers. To be precise, for each base point $x \in \mathcal{M}$ there is a sesquilinear metric $h_{x}: \mathcal{L}_{x} \times \mathcal{L}_{x} \rightarrow \mathbb{C}$ on the associated fiber $\mathcal{L}_{x}$. By convention, each $h_{x}$ is conjugate linear in the first argument. We will only consider finite-dimensional manifolds, $n:=\operatorname{dim}_{\mathbb{C}} \mathcal{M}<\infty$. Given a measure $m$ on $\mathcal{M}$ we may define an inner product

$$
(\psi, \phi):=\int_{\mathcal{M}} h(\psi, \phi) d m
$$

for sufficiently regular sections $\psi$ and $\phi$, where $h(\psi, \phi)$ is interpreted as the function $x \mapsto h_{x}(\psi(x), \phi(x))$.

Remark 2. In the definition of the inner product, $h$ and $m$ can be combined to a Hermitian-metric valued measure, hereafter denoted by $h \mathrm{~m}$. Indeed, this is a more appropriate way to view the definition, since the redundancy of rescaling $h$ while changing $m$ to compensate accordingly is manifest in the notation.

Definition 3. The linear space of sections in $\mathcal{L}$ will be denoted as $\Gamma_{\mathcal{L}}(\mathcal{M})$. The subspace of square-integrable sections on a complex line bundle $\mathcal{L}$ over a base manifold $\mathcal{M}$ is denoted by

$$
L^{2}(h m):=\left\{\psi \in \Gamma_{\mathcal{L}}(\mathcal{M}): \int_{\mathcal{M}} h(\psi, \psi) d m<\infty\right\} .
$$

When $\mathcal{L}$ is a holomorphic line bundle, we define the generalized Bergman space $L_{h o l}^{2}(h m)$ as the space of all holomorphic sections in $L^{2}(h m)$.

Remarks 4. Equipped with the previously defined inner product, the space $L^{2}(\mathrm{hm})$ containing all square-integrable sections becomes a Hilbert space in the usual way by identifying sections that differ up to sets of $\mathrm{hm}$-measure zero.

If $\mathcal{L}$ is a holomorphic line bundle and $m$, interpreted as a volume form, and $h$ are everywhere non-degenerate and smooth, then the generalized Bergman space $L_{h o l}^{2}(h m)$ is a space of functions that may be identified with a Hilbert-subspace of $L^{2}(h m)$. An outline of the completeness proof is given in Appendix A.

It may happen that $L_{h o l}^{2}(h m)$ only contains the zero section. Therefore, results about the dimensionality of this space are of fundamental interest. For the case of compact $\mathcal{M}$, see [9]. 
Lemma 5. Given a vector $u$ in a fiber above $x:=\pi(u)$, the point evaluation

$$
\begin{aligned}
\vartheta_{u}: L_{h o l}^{2}(h m) & \longrightarrow \mathbb{C} \\
\psi & \longmapsto h_{x}(u, \psi(x))
\end{aligned}
$$

defines a bounded linear functional, and by the Riesz representation theorem this evaluation can be realized as an inner product $\tilde{\psi}(u):=\left(e_{u}, \psi\right)=\vartheta_{u}(\psi)$ with a section $e_{u} \in L_{h o l}^{2}(h m)$. Two such sections form a kernel function $k(u, v):=\left(e_{u}, e_{v}\right)$ that is defined on $\mathcal{L} \times \mathcal{L}$ and sesquilinear in the fibers.

Proof. The detail that mostly deserves explanation is the boundedness of $\vartheta_{u}$. To verify this, we choose a local trivialization $\xi$ around the fiber generated by $u$, mapping $\pi^{-1}(U) \subset \mathcal{L}$, the subset of $\mathcal{L}$ above an open set $U$ to $V \times \mathbb{C}$, with an open ball $V \subset \mathbb{C}^{n}$ having the first component of $\xi(u)$ as the center.

Given a convergent sequence of sections $\left\{\psi^{(l)}\right\}_{l \in \mathbb{N}}$, we use as in Appendix A the mean value property of the associated holomorphic functions on $V$ to bound the value of $\vartheta_{u}\left(\psi^{(l)}\right)$ by a constant times the $L^{2}$-norm of $\psi^{(l)}$. Since the sequence has the Cauchy property, $\vartheta_{u}\left(\psi^{(l)}\right)$ is also Cauchy, and therefore convergent.

The sesquilinearity of $k$ results from the conjugate-linear scaling property $e_{c u}=$ $\bar{c} e_{u}$ for any $c \in \mathbb{C}$ and $u \in \mathcal{L}$.

Definition 6. A Schwartz kernel in a complex line bundle $\mathcal{L}$ is a family of linear mappings $\left\{\mathcal{S}(x, y): \mathcal{L}_{y} \rightarrow \mathcal{L}_{x}\right\}_{x, y \in \mathcal{M}}$, that is, $\mathcal{S}(x, y)$ is linear in vectors with base point $y$ and has as its values vectors at $x$. If $\mathcal{S}(x, y)$ is jointly continuous in $x$ and $y$, then it can be interpreted as continuous section in the bundle $\mathcal{L} \otimes \mathcal{L}^{*} \rightarrow \mathcal{M} \otimes \mathcal{M}$, where $\mathcal{L}^{*}$ is the dual bundle associating with each $x \in \mathcal{M}$ the space of complex linear forms on $\mathcal{L}_{x}$.

Proposition 7. The Schwartz kernel $K$ given in the terminology of the preceding lemma by $K(x, y) v=e_{v}(x)$ for $v \in \pi^{-1}(y)$ is jointly continuous in $x$ and $y$. We will call $K$ the reproducing kernel of $L_{h o l}^{2}(h m)$ because all $\psi \in L_{h o l}^{2}(h m)$ satisfy the identity

$$
\psi(x)=\int_{\mathcal{M}} K(x, y) \psi(y) d m(y)
$$

Proof. The joint continuity follows from the continuity of $e_{v}$ in $v$ and the uniform convergence of Cauchy sequences in $L_{h o l}^{2}(h m)$. These properties may be obtained using the definition of $e_{v}$ via (3) and the argument in Appendix A.

To derive (4), we consider in a first step the adjoint map $(K(x, y))^{*}: \pi^{-1}(x) \rightarrow$ $\pi^{-1}(y)$, in the usual way defined by $u \mapsto h_{x}(K(x, y) v, u) v$, independent of the choice of a normalized vector $v \in \pi^{-1}(y),\|v\|=1$. We claim that $(K(x, y))^{*}=$ $K(y, x)$, which means for all $u, v$ in fibers above $x$ and $y$, respectively, the equation $h_{x}(u, K(x, y) v)=h_{y}(K(y, x) u, v)$ holds. To simplify the following calculation, we assume that $u$ and $v$ are normalized; the general case follows by rescaling.

$$
\begin{aligned}
h_{x}(u, K(x, y) v) & =h_{x}\left(u, e_{v}(x)\right)=h_{x}\left(u, \tilde{e}_{v}(u) u\right) \\
& =\tilde{e}_{v}(u)=\overline{\tilde{e}_{u}(v)} \\
& =h_{y}\left(\tilde{e}_{u}(v) v, v\right)=h_{y}\left(e_{u}(y), v\right) \\
& =h_{y}(K(y, x) u, v)
\end{aligned}
$$


The second step for the derivation of (四) uses again a normalized vector $u$ above $x$,

$$
\begin{aligned}
\psi(x) & =\tilde{\psi}(u) u=\left(e_{u}, \psi\right) u=\int_{\mathcal{M}} h_{y}\left(e_{u}(y), \psi(y)\right) u d m(y) \\
& =\int_{\mathcal{M}} h_{y}(K(y, x) u, \psi(y)) u d m(y) \\
& =\int_{\mathcal{M}} h_{x}(u, K(x, y) \psi(y)) u d m(y) \\
& =\int_{\mathcal{M}} K(x, y) \psi(y) d m(y) .
\end{aligned}
$$

Comment 8. One of the goals in this work is to find a formula for this kernel. In principle, one could follow a Gram-Schmidt orthogonalization procedure, construct an orthonormal basis of sections $\left\{\eta_{l}\right\}_{l \in \mathbb{N}}$ and then express the reproducing kernel as a series $K(x, y)=\sum_{l} \eta_{l}(x) h_{y}\left(\eta_{l}(y), \cdot\right)$ that terminates after finite terms or converges uniformly on compact sets in $\mathcal{M} \times \mathcal{M}$. However, this procedure is too abstract to show how the geometry of $\mathcal{L}$ shapes the kernel. We will therefore present an alternative strategy, expressing $K$ in a probabilistic way.

Consequence 9. If $\mathrm{hm}$ is smooth and nowhere degenerate, then any bounded operator $B$ on $L_{h o l}^{2}(h m)$ possesses a sesqui-analytic integral kernel $B(x, y)$ that is characterized by the equation $h_{x}(u, B(x, y) v)=(K(\cdot, x) u, B K(\cdot, y) v)$, and the image of $\psi \in L_{h o l}^{2}(h m)$ is expressed as

$$
B \psi(x)=\int_{\mathcal{M}} B(x, y) \psi(y) d m(y) .
$$

Proof. That $B(x, y)$ is indeed an integral kernel results from the reproducing property (9) and Fubini's theorem. The sesqui-analyticity of $B(x, y)$ follows because the mapping $v \mapsto K(\cdot, \pi(v)) v=e_{v}$ into $L_{h o l}^{2}(h m)$ is antiholomorphic.

Remark 10. Since the right-hand side of equation (13) is defined even for $\psi \in$ $L^{2}(h m)$, any bounded operator extends naturally via its integral kernel to all of $L^{2}(h m)$. From this point of view, $K(x, y)$ is the integral kernel of an orthogonal projection operator, henceforth also called $K$, that maps $L^{2}(h m)$ onto $L_{h o l}^{2}(h m)$.

2.2. Berezin-Toeplitz Operators Defined via Quadratic Forms. In the remaining text, we assume that $h$ and $m$ are smooth and non-degenerate to ensure that $L_{h o l}^{2}(h m)$ is complete.

Definition 11. Given the Hilbert space $L_{h o l}^{2}(h m)$ and a real-valued function $f$ : $\mathcal{M} \rightarrow \mathbb{R}$, we consider the sesquilinear form

$$
\begin{aligned}
\mathcal{T}_{f}: \mathcal{Q}\left(\mathcal{T}_{f}\right) \times \mathcal{Q}\left(\mathcal{T}_{f}\right) & \longrightarrow \mathbb{C} \\
(\psi, \phi) & \longmapsto \int_{\mathcal{M}} f(x) h_{x}(\psi(x), \phi(x)) d m(x)
\end{aligned}
$$

with form domain

$$
\mathcal{Q}\left(\mathcal{T}_{f}\right):=\left\{\psi \in L_{h o l}^{2}(h m): \int_{\mathcal{M}}|f(x)| h_{x}(\psi(x), \psi(x)) d m(x)<\infty\right\} .
$$


When referring to $\mathcal{T}_{f}$ as a quadratic form, it is really the function $\psi \mapsto \mathcal{T}_{f}(\psi, \psi)$ that is meant.

Definition 12. Given a real-valued, bounded function $f: \mathcal{M} \rightarrow \mathbb{R}$, the form $\mathcal{T}_{f}$ specified in the preceding definition is bounded and symmetric. Therefore, it is associated with a self-adjoint operator $T_{f}$ satisfying $\left(\psi, T_{f} \psi\right)=\mathcal{T}_{f}(\psi, \psi)$ for all $\psi \in L_{h o l}^{2}(h m)$. In the context of generalized Bergman spaces, we call $T_{f}$ a selfadjoint Berezin-Toeplitz operator and the function $f$ its symbol.

Remarks 13. The original definition according to Berezin [1, 2] and its geometric interpretation by Cahen, Gutt, Rawnsley and others 9 13] do not refer to sesquilinear forms. Indeed, for bounded symbols the approach chosen here offers no new insights.

However, the use of sesquilinear forms is convenient for the construction of semibounded Berezin-Toeplitz operators described in the remaining part of this section. The implicit goal is to find a large class of possibly unbounded symbols $f$ that lead to closed, semibounded quadratic forms $\mathcal{T}_{f}$ and thus yield unique self-adjoint Berezin-Toeplitz operators $T_{f}$ via the Friedrichs construction characterized by equation (19). In fact, this goal leads the discussion from abstract conditions ensuring the semiboundedness of $T_{f}$ to a more concrete class of admissible symbols presented in the next section.

Lemma 14. If the form $\mathcal{T}_{f^{+}}$belonging to the positive part $f^{+}: x \mapsto \max \{f(x)$, $0\}$ of a function $f: \mathcal{M} \rightarrow \mathbb{R}$ is densely defined and the negative part $f^{-}: x \mapsto$ $\max \{-f(x), 0\}$ can be incorporated in $\mathcal{T}_{f}$ as a form-bounded perturbation, meaning

$$
\mathcal{T}_{f^{-}}(\psi, \psi) \leq c_{1} \mathcal{T}_{f^{+}}(\psi, \psi)+c_{2}\|\psi\|^{2}
$$

with a relative form bound $c_{1}<1$ and a constant $c_{2} \geq 0$, then $\mathcal{T}_{f}$ is closed on $\mathcal{Q}\left(\mathcal{T}_{f}\right)=\mathcal{Q}\left(\mathcal{T}_{f^{+}}\right)$and has a lower bound $c \in \mathbb{R}$, such that $\mathcal{T}_{f}(\psi, \psi) \geq c\|\psi\|^{2}$.

Proof. The first part of the proof is to show that the sesquilinear form belonging to a non-negative function $f \geq 0$ is closed, in other words, we need to show that $\mathcal{Q}\left(\mathcal{T}_{f}\right)$, equipped with the form-norm $\|\bullet\|_{\mathcal{T}_{f}}$ defined by

$$
\|\psi\|_{\mathcal{T}_{f}}:=\left(\mathcal{T}_{f}(\psi, \psi)+\|\psi\|^{2}\right)^{1 / 2} \text { for } \psi \in \mathcal{Q}\left(\mathcal{T}_{f}\right)
$$

is complete.

Suppose $\left(\psi_{l}\right)_{l \in \mathbb{N}}$ is a Cauchy sequence with respect to the form-norm. Due to the estimate $\|\psi\| \leq\|\psi\|_{\mathcal{T}_{f}}$ the sequence is convergent in $L_{h o l}^{2}(\mathrm{hm}), \psi_{l} \rightarrow \psi$. Using pointwise convergence and Fatou's lemma, we have $\left\|\psi-\psi_{l}\right\|_{\mathcal{T}_{f}} \leq \liminf \inf _{k \rightarrow \infty}\left\|\psi_{k}-\psi_{l}\right\|_{\mathcal{T}_{f}}$ and therefore the sequence $\left(\psi_{l}\right)_{l \in \mathbb{N}}$ converges with respect to the form-norm.

The remaining part of the proof is the so-called KLMN theorem, see [32] or [33, Theorem X.17]. It goes back to works of Kato [34], Lax and Milgram [35, Lions [36] and Nelson [37.

Proposition 15. If the form $\mathcal{T}_{f}$ is closed and has the greatest lower bound $c \in \mathbb{R}$, then it belongs to a unique self-adjoint operator $T_{f}$ that is characterized in terms of the square-root $\sqrt{T_{f}-c}$ satisfying

$$
\left(\sqrt{T_{f}-c} \phi, \sqrt{T_{f}-c} \psi\right)+c(\phi, \psi)=\mathcal{T}_{f}(\phi, \psi)
$$

for all $\phi$ and $\psi$ in the domain $\mathcal{D}\left(\sqrt{T_{f}-c}\right)=\mathcal{Q}\left(\mathcal{T}_{f}\right)$. 
Proof. Again, we refer to the literature [38, Theorem VIII.15] or [39, Theorem 5.36] for the proof of this result which we call the Friedrichs construction.

Remarks 16. As a special case of Consequence 9, when $f$ is a bounded function, $T_{f}$ has an integral kernel $T_{f}(x, y)$ characterized by $h_{x}\left(u, T_{f}(x, y) v\right)=(K(\cdot, x) u$, $f K(\cdot, y) v)$, where $u, v \in \mathcal{L}$ have base points $x$ and $y$, and the scalar product is taken in $L^{2}(h m)$.

For $\psi \in \mathcal{D}_{\text {min }}\left(T_{f}\right):=\left\{\psi \in L_{h o l}^{2}(h m), f \psi \in L^{2}(h m)\right\}$, the identity $T_{f} \psi=K(f \psi)$ relates $T_{f}$ to the traditional way of defining a Berezin-Toeplitz operator as a composition of a multiplication operator with the orthogonal projection $K$. However, it may happen that $\mathcal{D}_{\min }\left(T_{f}\right)$ does not include all of $L_{h o l}^{2}(h m)$, although the operator $T_{f}$ is bounded.

A disadvantage of defining $T_{f}$ by a semibounded form is that in general, nothing is known about its domain. The situation is different if a domain of essential selfadjointness can be identified for $T_{f}$. Such situations have been investigated in detail 40, 41] for the case of the so-called Fock-Bargmann space.

The definition of Berezin-Toeplitz operators clearly does not rely on the validity of a correspondence principle, and we will also not need to refer to it hereafter. Because of its physical importance, we mention that in the special setting of holomorphic line bundles over homogeneous or compact Kähler manifolds, the Berezin-Toeplitz operators defined on the Hilbert space $L_{h o l}^{2}(h m)$, with $m$ being the Liouville form associated with the bundle curvature, are known to observe a correspondence principle, see [2, 17] or [9, 18]. Moreover, in the compact case the same kind of classical asymptotics can be proved for more general almost-complex manifolds [42].

\section{Self-Adjoint Berezin-Toeplitz Operators as Monotone Limits of SEmibounded Schrödinger Operators}

The main motivation for this section is to relate Berezin-Toeplitz and Schrödinger operators. An important application concerns the transfer of well-known self-adjointness criteria to the setting of Berezin-Toeplitz operators. In this section and the following one we derive conditions that are more accessible than verifying the abstract form-boundedness of $T_{f^{-}}$with respect to $T_{f^{+}}$according to inequality (17).

At first, the Riemannian structure seems to be an auxiliary element that is not needed in the definition of Berezin-Toeplitz operators according to the prescription of the preceding section. However, continuing the line of thought in the introductory remarks given there, we note that due to the coherent-state embedding $x \mapsto e(x):=$ $\left\{e_{u}: u \in \mathcal{L}_{x}\right\}$ the inner product of the Hilbert space provides a natural metric on $T \mathcal{L}$, which by its invariance under scalar multiplication in the fibers passes as Fubini-Study type metric [43, Appendix 3] to the tangent bundle TM. It is straightforward to check that the imaginary, skew-symmetric part of the FubiniStudy metric is closed, which makes $\mathcal{M}$ a Kähler manifold. The real part of this metric can then be used to define a Riemannian structure. In short, a Riemannian metric is present as a consequence of the quantization prescription.

In the following, we consider Hilbert spaces $L_{h o l}^{2}(\mathrm{hm})$ of square-integrable holomorphic sections in a holomorphic Hermitian line bundle $\mathcal{L}$ that has a base manifold $\mathcal{M}$ with a Kähler metric. A priori, the natural volume measure $m$ associated with 
the real part of the Kähler metric need not be in a prequantum relation (33) with the curvature of $\mathcal{L}$.

\subsection{Bochner's Laplacian and its Relation to the Holomorphic Laplacian.}

Several Laplacians will be introduced in this section, each one is characterized by an associated positive definite quadratic form. Later, Schrödinger operators will arise from perturbations of these forms.

Conventions 17. By default, $\mathcal{M}$ is always a $d$-dimensional Riemannian manifold, and whenever it appears in conjunction with the holomorphic line bundle $\mathcal{L}$, it is tacitly understood to be the base manifold, with $d=2 n$. The Hermitian metric $h$ on $\mathcal{L}$ and the natural volume measure $m$ on $\mathcal{M}$ are as forms assumed to be smooth and non-degenerate. The class of smooth vector fields that are at each point realvalued differential operators on real-valued, smooth functions is written as $\Upsilon_{\mathbb{R}}(\mathcal{M})$. The complexified version is written as $\Upsilon(\mathcal{M})$. Whenever a smooth vector field $Y$ given on an open set $U \subset \mathcal{M}$ vanishes on all antiholomorphic functions in $U$, we write $Y \in \Upsilon^{(1,0)}(U)$, and if this happens for all holomorphic ones $Y \in \Upsilon^{(0,1)}(U)$. We will not distinguish between a Riemannian metric $g$ on the tangent bundle $T \mathcal{M}$ and its sesquilinear extension to the complexified tangent bundle $T^{\mathbb{C}} \mathcal{M}$, as usual conjugate linear in the first argument. Similarly, the Levi-Civita connection Cov is made complex linear on $T^{\mathbb{C}} \mathcal{M} \times T^{\mathbb{C}} \mathcal{M}$, and the divergence div is thus defined as the trace $\operatorname{Tr}^{T \mathcal{M}} b_{Y}$ for all $Y \in \Upsilon(\mathcal{M})$ with the sesquilinear form given by $b_{Y}:(X, Z) \mapsto\left(X, \operatorname{Cov}_{Z} Y\right)$. The gradient of a function $f$ is a vector field denoted as $\operatorname{grad} f$.

With a view to Lemma 19, from now on all manifolds are tacitily assumed to be pathwise connected.

Definition 18. The operator obtained by the Friedrichs construction corresponding to the closure of the quadratic form

$$
\mathcal{E}(f, f):=\int_{\mathcal{M}} g(\operatorname{grad} f, \operatorname{grad} f) d m
$$

with initial form domain $C_{c}^{\infty}(\mathcal{M})$ is called the negative Dirichlet Laplacian $-\Delta$ on $L^{2}(m)$.

Suppose $\mathcal{L}$ is a Hermitian line bundle with a compatible connection $\nabla$. The negative Bochner Laplacian $-\Delta^{\mathcal{L}}$ on $L^{2}(\mathrm{hm})$ arises via the Friedrichs construction from

$$
\mathcal{E}^{\mathcal{L}}(\psi, \psi):=\int_{\mathcal{M}} \operatorname{Tr}^{T \mathcal{M}} h(\nabla \psi, \nabla \psi) d m
$$

defined on $C_{c \mathcal{L}}^{\infty}(\mathcal{M})$, the space of smooth sections with compact support. Hereby, the trace operation is defined as before by choosing an orthonormal basis $\left\{E_{k}\right\}_{k=1}^{d}$ in each $T_{x} \mathcal{M}$ such that $\operatorname{Tr}^{T \mathcal{M}} h(\nabla \psi, \nabla \psi)=\sum_{k=1}^{d} h\left(\nabla_{E_{k}} \psi, \nabla_{E_{k}} \psi\right)$.

Lemma 19. Every complete Riemannian manifold $\mathcal{M}$ admits a localizing sequence of smooth cut-off functions with a uniformly attenuated gradient bound. This means, there is an increasing sequence $\left\{\eta_{l}\right\}_{l \in \mathbb{N}}$ of smooth functions $\eta_{l}$ pointwise converging to unity, $\eta_{l}(x) \nearrow 1$ for all $x \in \mathcal{M}$, each $\eta_{l}$ has compact support, and the uniform gradient bound $g\left(\operatorname{grad} \eta_{l}\right.$, grad $\left.\eta_{l}\right) \leq C_{l}$ holds for some sequence $\left\{C_{l}\right\}_{l \in \mathbb{N}}$ of positive numbers $C_{l} \geq 0$ converging to zero. 
Proof. The construction uses a result by Greene and Wu 144 , Corollary to Proposition 2.1], by which one may approximate the distance from a fixed point $y \in \mathcal{M}$ with a smooth function. To be precise, one obtains a smooth function $v: \mathcal{M} \rightarrow \mathbb{R}$ such that $\|\operatorname{grad} v\|<1$ and $|v(x)-\operatorname{dist}(x, y)|<1$ for all $x \in \mathcal{M}$.

For the construction of the cut-off functions, we pick a real-valued smooth function $\eta: \mathbb{R} \rightarrow[0,1]$ that is bounded above and below by characteristic functions $\chi_{[-1,1]} \leq \eta \leq \chi_{[-2,2]}$, ensuring compact support in the interval $[-2,2]$. The composition $\eta_{l}(x):=\eta\left(\frac{1}{2^{2}} v(x)\right)$ then defines an increasing sequence of smooth functions $\eta_{l} \nearrow 1$ with the gradient bound

$$
\operatorname{grad} \eta_{l}=\frac{1}{2^{l}} \eta^{\prime}\left(\frac{1}{2^{l}} v(x)\right) \operatorname{grad} v(x) \leq \frac{1}{2^{l}} \max _{r \in \mathbb{R}}\left|\eta^{\prime}(r)\right| .
$$

In addition, due to the completeness of the manifold, the support of each $\eta_{l}$ is compact since it is contained in the closed set $v^{-1}\left(\left[-2^{l}, 2^{l}\right]\right)$.

Theorem 20. If the Riemannian manifold $\mathcal{M}$ is complete, then $-\Delta$ is essentially self-adjoint on $C_{c}^{\infty}(\mathcal{M})$. The same holds for $-\Delta^{\mathcal{L}}$ in a Hermitian line bundle $\mathcal{L}$ with a compatible connection $\nabla$. Moreover, $-\Delta^{\mathcal{L}}$ has $C_{c \mathcal{L}}^{\infty}(\mathcal{M})$ as a domain of essential self-adjointness.

Proof. It is sufficient to show this for $\Delta^{\mathcal{L}}$, since $\Delta$ can be considered as the Bochner Laplacian on the trivial bundle $\mathcal{M} \times \mathbb{C}$ with the obvious Hermitian structure. We adapt Davies' treatment of the Dirichlet Laplacian [45, Theorem 5.2.3] in combination with the localizing sequence of cut-off functions described in the preceding construction.

The essential self-adjointness of $-\Delta^{\mathcal{L}}$ is by its positivity equivalent 33 , Theorem X.26] to having only the zero vector in the orthogonal complement of $\left(-\Delta^{\mathcal{L}}+\right.$ 1) $C_{c \mathcal{L}}^{\infty}(\mathcal{M})$.

Suppose there is a nonzero vector $u \perp\left(-\Delta^{\mathcal{L}}+1\right) C_{c \mathcal{L}}^{\infty}(\mathcal{M})$, in other words the equation $\Delta^{\mathcal{L}} u=u$ has a weak solution $u \in L^{2}(h m)$. Using the localizing sequence $\left\{\eta_{l}\right\}_{l \in \mathbb{N}}$ described above, we may estimate

$$
\begin{aligned}
0 & \geq-\left\|\eta_{l} u\right\|_{2}^{2}=\mathcal{E}^{\mathcal{L}}\left(\eta_{l}^{2} u, u\right)=\int_{\mathcal{M}} \sum_{k=1}^{d} h\left(\nabla_{k} \eta_{l}^{2} u, \nabla_{k} u\right) d m \\
& =\int_{\mathcal{M}} \sum_{k=1}^{d} 2 \eta_{l} E_{k}\left(\eta_{l}\right) h\left(u, \nabla_{k} u\right) d m+\int_{\mathcal{M}} \sum_{k=1}^{d} \eta_{l}^{2} h\left(\nabla_{k} u, \nabla_{k} u\right) d m .
\end{aligned}
$$

The last term is positive and we conclude that it must be bounded by

$$
\begin{aligned}
\int_{\mathcal{M}} \sum_{k=1}^{d} \eta_{l}^{2} h\left(\nabla_{k} u, \nabla_{k} u\right) d m & \leq 2 \int_{\mathcal{M}} \sum_{k=1}^{d} \eta_{l}\left|E_{k}\left(\eta_{l}\right)\right|\left|h\left(u, \nabla_{k} u\right)\right| d m \\
& \leq 2 \int_{\mathcal{M}} \sum_{k=1}^{d} \eta_{l}\left|E_{k}\left(\eta_{l}\right)\right| \sqrt{h(u, u) h\left(\nabla_{k} u, \nabla_{k} u\right)} d m \\
& \leq 2 \int_{\mathcal{M}} \eta_{l}\left\|\operatorname{grad} \eta_{l}\right\|_{\infty} \sqrt{h(u, u) \sum_{k} h\left(\nabla_{k} u, \nabla_{k} u\right)} d m
\end{aligned}
$$


where the Cauchy-Schwarz inequality has been used repeatedly. With the abbreviation $c_{l}:=\eta_{l} \sqrt{\sum_{k} h\left(\nabla_{k} u, \nabla_{k} u\right)}$, we obtain

$$
\int_{\mathcal{M}} c_{l}^{2} d m \leq 2 \int_{\mathcal{M}} c_{l}\left\|\operatorname{grad} \eta_{l}\right\|_{\infty} \sqrt{h(u, u)},
$$

and after using the Cauchy-Schwarz inequality again,

$$
\left\|c_{l}\right\|_{2}^{2} \leq 2\left\|c_{l}\right\|_{2}\left\|\operatorname{grad} \eta_{l}\right\|_{\infty}\|u\|_{2}
$$

To avoid confusion, $\|\cdot\|_{2}$ denotes the $L^{2}$-norm and the term $\left\|\operatorname{grad} \eta_{l}\right\|_{\infty}$ the essential supremum of the Riemannian length of $\operatorname{grad} \eta_{l}(x)$ over $x \in \mathcal{M}$. This last inequality involves finite quantities on both sides, because $u$ is a smooth function by an argument related to Sobolev norms as in Appendix B. The properties of the localizing sequence $\left\{\eta_{l}\right\}$ imply that the right-hand side approaches zero in the limit $l \rightarrow \infty$. Therefore, by Fatou's lemma $\mathcal{E}^{\mathcal{L}}(u, u)=0$ or $\nabla u=0$, which is in contradiction to the assumption $\Delta^{\mathcal{L}} u=u \neq 0$.

Now we investigate the interplay between Riemannian and complex structures on $\mathcal{M}$. The fundamental ingredient is the assumption that the connection $\nabla$ is compatible with the Hermitian metric and the holomorphic structure, that is, $\nabla_{X} \psi=0$ for all locally antiholomorphic vector fields $X$ and locally holomorphic sections $\psi$.

Definition 21. Suppose we pick a local section in the orthonormal frame bundle of $T^{(0,1)} \mathcal{M}$, which means in a sufficiently small open set $U \subset \mathcal{M}$, we have antiholomorphic vector fields $\bar{Z}_{1}, \bar{Z}_{2}, \ldots \bar{Z}_{d / 2} \in \Upsilon^{(0,1)}(U)$ that are orthonormal, $g\left(\bar{Z}_{k}, \bar{Z}_{l}\right)=\delta_{k l}$. For a section $\psi$, the value of the antiholomorphic trace $\operatorname{Tr}^{(0,1)} h(\nabla \psi, \nabla \psi):=\sum_{k} h\left(\nabla_{\bar{Z}_{k}} \psi, \nabla_{\bar{Z}_{k}} \psi\right)$ depends on the metric and the connection $\nabla$, not on the particular choice of orthonormal antiholomorphic vector fields. Therefore, we may define the negative holomorphic Laplacian $-\Delta^{(0, \bullet)}$, in a manner analogous to the previous definitions as the operator corresponding to the closure of the quadratic form

$$
\mathcal{E}^{(0, \bullet)}(\psi, \psi):=\int_{\mathcal{M}} \operatorname{Tr}^{(0,1)} h(\nabla \psi, \nabla \psi) d m
$$

initially defined on sections $\psi$ in the domain $C_{c \mathcal{L}}^{\infty}(\mathcal{M})$.

Remark 22. Let $g$ be a Riemannian metric on a complex manifold $\mathcal{M}$ and Cov its Levi-Civita connection. It is straightforward to check in local coordinates [31, Proposition 7.14] that $g$ is the real part of a Kähler metric if and only if it is compatible with the almost complex structure $J$ and if Cov preserves the splitting of $\Upsilon(\mathcal{M})$ into holomorphic and antiholomorphic parts, that is, $\operatorname{Cov}_{X} J Y=J \operatorname{Cov}_{X} Y$ for all $X, Y \in \Upsilon(\mathcal{M})$.

Proposition 23. Let $g$ be the real part of a Kähler metric on the the $d$-dimensional base manifold $\mathcal{M}$ of a holomorphic line bundle $\mathcal{L}$, and assume the Bochner and holomorphic Laplacians are defined as above. Then a Weitzenböck-type formula relates both Laplacians

$$
\Delta^{(0, \bullet)}=\frac{1}{2}\left(\Delta^{\mathcal{L}}-\rho\right)
$$

with a zeroth-order term $\rho$. Given an antiholomorphic orthonormal frame $\left\{\bar{Z}_{k}\right\}_{k=1}^{d / 2}$ of $T^{(0,1)} \mathcal{M}$, the term $\rho$ is expressed as $\rho(x) \psi(x)=\sum_{k=1}^{d / 2} R_{\bar{Z}_{k}, Z_{k}} \psi(x)$. 
Proof. The first step of the proof is to identify $-\Delta^{(0, \bullet)}$ and $\Delta^{\mathcal{L}}$ as differential operators when acting on a smooth, compactly supported section $\psi \in C_{c \mathcal{L}}^{\infty}(\mathcal{M})$. According to the usual derivation 447 we find

$$
\Delta^{\mathcal{L}} \psi=\sum_{k=1}^{d} \nabla_{E_{k}} \nabla_{E_{k}} \psi-\nabla_{\operatorname{Cov}_{E_{k}} E_{k}} \psi
$$

and

$$
\Delta^{(0, \bullet)} \psi=\sum_{k=1}^{d / 2}\left(\nabla_{Z_{k}} \nabla_{\bar{Z}_{k}}-\nabla_{\operatorname{Cov}_{Z_{k}} \bar{Z}_{k}}\right) \psi .
$$

Here, $\left\{E_{k}, J E_{k}\right\}_{k=1}^{d / 2}$ is a local orthonormal frame in $T \mathcal{M}$ and the antholomorphic frame $\left\{\bar{Z}_{k}\right\}_{k=1}^{d / 2}$ is obtained via $\bar{Z}_{k}=\frac{1}{\sqrt{2}}\left(E_{k}+i J E_{k}\right) \in T^{(0,1)} \mathcal{M}$. The derivation relies on the compatibility of the connection $\nabla$ with the Hermitian metric, the resolution of the identity $\sum_{k=1}^{d / 2}\left(\left(\cdot, E_{k}\right) E_{k}+\left(\cdot, J E_{k}\right) J E_{k}\right)=\operatorname{id}_{T \mathcal{M}}$ and the compatibility between the almost complex structure $J$ and the Levi-Civita connection Cov. Now the claimed relationship (30) follows from (31) and (32) with the torsion-free property of the Levi-Civita connection Cov that implies $\left[\bar{Z}_{k}, Z_{k}\right]=\bar{Z}_{k} Z_{k}-Z_{k} \bar{Z}_{k}=$ $\operatorname{Cov}_{\bar{Z}_{k}} Z_{k}-\operatorname{Cov}_{Z_{k}} \bar{Z}_{k}$.

Remark 24. If the curvature $R$ of the bundle and the Kähler form $\omega=\frac{1}{2} g(\cdot, J \cdot)$ are in the prequantum relation

$$
R_{X, Y}=\frac{i}{\hbar} \omega(X, Y)
$$

for any $X, Y \in \Upsilon(\mathcal{M})$, then $\rho$ is a constant,

$$
\rho=i \sum_{k=1}^{d / 2} R_{E_{k}, J E_{k}}=-\frac{1}{2 \hbar} \sum_{k=1}^{d / 2} g\left(E_{k}, E_{k}\right)=-\frac{d}{4 \hbar} .
$$

3.2. Berezin-Toeplitz Operators as Limits of Schrödinger Operators. This subsection shows how a Berezin-Toeplitz operator can be extended to a family of Schrödinger operators and be reconstructed as a monotone limit of this family. A major benefit is that the knowledge about Schrödinger operators may be used to find sufficient conditions for the semiboundedness of $\mathcal{T}_{f}$, thereby ensuring the self-adjointness of the associated Berezin-Toeplitz operator.

Convention 25. In the following, $\mathcal{L}$ is always a holomorphic Hermitian line bundle and $g$ is assumed to be the real part of a Kähler metric on the $d$-dimensional base manifold $\mathcal{M}$.

Proposition 26. If the manifold $\mathcal{M}$ is complete, then the space $L_{h o l}^{2}(h m)$ is in the domain of the form-closure of $\mathcal{E}^{(0, \bullet)}$ and can be identified as the null-space $\left\{\psi \in L^{2}(h m):-\Delta^{(0, \bullet)} \psi=0\right\}$ of the holomorphic Laplacian $-\Delta^{(0, \bullet)}$.

Proof. Given $\psi \in L_{h o l}^{2}(h m)$, we need to construct a Cauchy sequence $\left\{\psi_{l}\right\}_{l \in \mathbb{N}}$ in $C_{c \mathcal{L}}^{\infty}(\mathcal{M})$ which converges to $\psi$ with respect to the form-norm, $\left\|\psi_{l}-\psi\right\|_{\mathcal{E}^{(0, \bullet} \bullet} \rightarrow$ 0 . To this end, we use an increasing sequence of localizing cut-off functions $\eta_{l}$ : $\mathcal{M} \rightarrow[0,1]$ observing the uniform gradient bound $\sup _{x \in \mathcal{M}}\left\|\operatorname{grad} \eta_{l}(x)\right\| \leq \frac{C}{2^{l}}$ for some constant $C>0$, as described in the preceding part of this section. Then by 
monotone convergence $\left\|\eta_{l} \psi-\psi\right\| \rightarrow 0$, and the remaining term in the form-norm can be estimated by

$$
\begin{aligned}
\mathcal{E}^{(0, \bullet)}\left(\eta_{l} \psi, \eta_{l} \psi\right)= & \int_{\mathcal{M}} \sum_{k} h\left(\nabla_{\bar{Z}_{k}} \eta_{l} \psi, \nabla_{\bar{Z}_{k}} \eta_{l} \psi\right) d m \\
= & \sum_{k} \int_{\mathcal{M}}\left(\left|Z_{k}\left(\bar{\eta}_{l}\right)\right|^{2} h(\psi, \psi)+\left|\eta_{l}\right|^{2} h\left(\nabla_{\bar{Z}_{k}} \psi, \nabla_{\bar{Z}_{k}} \psi\right)\right. \\
& \left.\quad+2 \Re\left(Z_{k}\left(\bar{\eta}_{l}\right) h\left(\psi, \nabla_{\bar{Z}_{k}} \psi\right)\right)\right) d m \\
\leq & \frac{C^{2}}{2^{2 l}}\|\psi\|^{2}+\int_{\mathcal{M}}\left|\eta_{l}\right|^{2} \sum_{k} h\left(\nabla_{\bar{Z}_{k}} \psi, \nabla_{\bar{Z}_{k}} \psi\right) d m \\
& +2 \int_{\mathcal{M}} \sum_{k}\left|Z_{k}\left(\bar{\eta}_{l}\right) h\left(\psi, \nabla_{\bar{Z}_{k}} \psi\right)\right| d m .
\end{aligned}
$$

Using the Cauchy-Schwarz inequality, we have

$$
\mathcal{E}^{(0, \bullet)}\left(\eta_{l} \psi, \eta_{l} \psi\right) \leq \frac{C^{2}}{2^{2 l}}\|\psi\|^{2}+\mathcal{E}^{(0, \bullet)}(\psi, \psi)+\frac{2 C}{2^{l}}\|\psi\|\left(\mathcal{E}^{(0, \bullet)}(\psi, \psi)\right)^{1 / 2}
$$

so by dominated convergence $\mathcal{E}^{(0, \bullet)}\left(\eta_{l} \psi-\psi, \eta_{l} \psi-\psi\right) \rightarrow 0$. Thus, both terms in the form-norm converge to zero.

Definition 27. A semibounded Schrödinger operator $S_{D, q}^{\mathcal{L}}$ on $L^{2}(h m)$ is the selfadjoint operator associated with the form

$$
\mathfrak{S}_{D, q}^{\mathcal{L}}(\psi, \psi)=D \mathcal{E}^{\mathcal{L}}(\psi, \psi)+(\psi, q \psi),
$$

where $D>0$ is some coupling constant and the requirement

$$
\left(\psi, q^{-} \psi\right) \leq c_{1} \mathfrak{S}_{D, q^{+}}^{\mathcal{L}}(\psi, \psi)+c_{2}(\psi, \psi)
$$

is satisfied with relative form bound $c_{1}<1$ and some constant $c_{2} \geq 0$. Thus, the form domain of $\mathfrak{S}_{D, q}^{\mathcal{L}}$ is obtained from the closure of $C_{c \mathcal{L}}^{\infty}(\mathcal{M}) \cap\left\{\psi:\left(\psi, q^{+} \psi\right)<\infty\right\}$.

Remark 28. If in addition to the requirement (40) the curvature term $\rho$ of Proposition 23 is also a form-bounded perturbation of $S_{q^{+}}^{\mathcal{L}}$, then

$$
\mathfrak{S}_{D, q}^{(0, \bullet)}: \psi \mapsto \mathfrak{S}_{D, D \rho+q}^{\mathcal{L}}(\psi, \psi)=D \mathcal{E}^{(0, \bullet)}(\psi, \psi)+(\psi, q \psi)
$$

has the same form domain as $\mathfrak{S}_{D, q}^{\mathcal{L}}$ and also defines a generalized Schrödinger operator, hereafter referred to as $S_{D, q}^{(0, \bullet)}$.

Theorem 29. If the assumptions of Proposition 20 and Remark 28 are fulfilled, then $\mathfrak{S}_{D, f}^{(0, \bullet)}$ is semibounded and $\mathcal{T}_{f}$ on $L_{h o l}^{2}(h m)$ is closed and semibounded.

Proof. First we note $\mathfrak{S}_{D, f}^{(0, \bullet)}(\psi, \psi)=\mathcal{T}_{f}(\psi, \psi)$ for any $D>0$ and $\psi \in \mathcal{Q}\left(\mathcal{T}_{f}\right)$. Thus, we only need to show that the restriction of $\mathfrak{S}_{D, f}^{(0, \bullet)}$ to the closed subspace $L_{h o l}^{2}(h m)$ is again a closed and semibounded form.

To show closedness, assume a sequence $\left(\psi_{l}\right)_{l \in \mathbb{N}}$ in $L_{h o l}^{2}(h m)$ which is Cauchy with respect to the form-norm. Then by the closedness of $\mathfrak{S}_{D, f}^{(0, \bullet)}$ the sequence has a limit $\psi \in \mathcal{Q}\left(\mathfrak{S}_{D, f}^{(0, \bullet)}\right)$. However, this limit is contained in $L_{h o l}^{2}(h m)$, because the sequence $\left(\psi_{l}\right)_{l \in \mathbb{N}}$ also converges with respect to the usual norm on $L_{h o l}^{2}(h m)$. 
Semiboundedness follows from the inequality

$$
\inf _{\substack{\psi \in L^{2}(h m),\|\psi\|=1}} \mathfrak{S}_{D, f}^{(0, \bullet)}(\psi, \psi) \leq \inf _{\substack{\psi \in L_{h o l}^{2}(h m) \\\|\psi\|=1}} \mathfrak{S}_{D, f}^{(0, \bullet)}(\psi, \psi)
$$

due to the set inclusion $L_{h o l}^{2}(h m) \subset L^{2}(h m)$.

Remarks 30. As stated, the above theorem does not imply that $\mathcal{T}_{f}$ is densely defined. Therefore, $T_{f}$ might be self-adjoint only on a Hilbert-subspace of $L_{h o l}^{2}(\mathrm{hm})$.

In analogy with Lemma 14, it is sufficient for the closedness and semiboundedness of $\mathcal{T}_{f}$ when for some $D>0$ the negative part $f^{-}$can be incorporated as a formbounded perturbation of $\mathfrak{S}_{D, f^{+}}^{(0, \bullet)}$ with relative form bound strictly less than one. However, this condition is not as easy to characterize in terms of $f$ as the stronger assumption in the preceding theorem.

Consequence 31. If the assumptions of the preceding theorem hold, then the semigroup generated by $S_{D, f}^{(0, \bullet)}$ converges in the limit $D \rightarrow \infty$ strongly to a BerezinToeplitz semigroup,

$$
\lim _{D \rightarrow \infty} e^{-t S_{D, f}^{(0, \bullet)}} \psi=e^{-t T_{f}} K_{f} \psi,
$$

where $t>0, \psi \in L^{2}(h m)$, and the orthogonal projector $K_{f}=K_{f}^{*} K_{f}$ maps onto the closure of $\mathcal{Q}\left(\mathcal{T}_{f}\right)$ in $L_{\text {hol }}^{2}(\mathrm{hm})$.

Proof. The limit $D \rightarrow \infty$ of $\mathfrak{S}_{D, f}^{(0, \bullet)}$ yields a non-densely defined quadratic form

$$
\mathfrak{S}_{\infty, f}^{(0, \bullet)}: \psi \mapsto \lim _{D \rightarrow \infty} \mathfrak{S}_{D, f}^{(0, \bullet)}(\psi, \psi)
$$

which is by inspection identical with $\mathcal{T}_{f}$.

The monotone convergence implies then that $\mathcal{T}_{f}$ is closed [48] and the semiboundedness follows from that of $\mathfrak{S}_{D, f}^{(0, \bullet)} \leq \mathcal{T}_{f}$ for some $D>0$. These properties imply that the Berezin-Toeplitz operator $T_{f}$ associated with $\mathcal{T}_{f}$ is self-adjoint on the closure $\overline{\mathcal{Q}\left(\mathcal{T}_{f}\right)} \subset L_{h o l}^{2}(h m)$. Again by the monotone convergence of forms the selfadjoint operators $S_{D, f}^{(0, \bullet)}$ converge in the strong resolvent sense 48], which in turn implies strong convergence of the semigroups they generate [38, Theorem S.14].

\section{Probabilistic Representation of Berezin-Toeplitz Semigroups}

This section introduces a new element into the discussion of Berezin-Toeplitz operators, the concept of Brownian motion on the base manifold $\mathcal{M}$ of the holomorphic line bundle $\mathcal{L}$. In terms of this stochastic process, one may characterize the Kato class of functions on $\mathcal{M}$. It turns out that Kato decomposable functions $f$ lead to semibounded, self-adjoint Berezin-Toeplitz operators $T_{f}$ on $L_{h o l}^{2}(h m)$, where $m$ is the Riemannian volume measure on $\mathcal{M}$. The final result in this section is a probabilistic expression for Berezin-Toeplitz semigroups, referred to as the Daubechies-Klauder formula. It is convenient that the validity of the DaubechiesKlauder formula is expressed in terms of the Kato class associated with Brownian motion and thus the admissibility of bundle curvature and classical Hamiltonian in the quantization procedure and path-integral formulation are intrinsically characterized by the underlying geometry. 
4.1. The Kato Class and a Version of the Feynman-Kac Formula. We adopt the usual terminology: An amost surely continuous process B with values in the Riemannian manifold $\mathcal{M}$ is called Brownian motion with diffusion constant $D>0$ if for every smooth function $\phi \in C^{\infty}(\mathcal{M})$, the difference

$$
\mathrm{M}_{t}:=\phi \circ \mathrm{B}_{t}-\phi \circ \mathrm{B}_{0}-\int_{0}^{t} D \Delta \phi \circ \mathrm{B}_{s} d s
$$

is a real-valued continuous local martingale $\mathrm{M}$.

A probability measure governing Brownian motion with diffusion constant $D>0$ and almost surely fixed starting point $\mathrm{B}_{0}=x \in \mathcal{M}$ will be denoted as $\mathbb{P}_{x}^{D}$. The expectation with respect to this probability measure is written as $\mathbb{E}_{x}^{D}$.

A Riemannian manifold $\mathcal{M}$ is called Brownian complete if for a fixed diffusion constant $D>0$, a Brownian motion B starting at any $x \in \mathcal{M}$ has an infinite explosion time.

Definition 32. Let $\mathcal{M}$ be a Brownian-complete Riemannian manifold with a family of Brownian-motion measures $\left\{\mathbb{P}_{x}^{D}\right\}_{x \in \mathcal{M}}$ having a fixed diffusion constant $D>0$. A real-valued function $q: \mathcal{M} \rightarrow \mathbb{R}$ belongs to the Kato class $\mathcal{K}\left(\mathbb{P}^{D}\right)$ if the following condition is satisfied:

$$
\lim _{t \searrow 0} \sup _{x \in \mathcal{M}} \int_{0}^{t} \mathbb{E}_{x}^{D}\left[|q|\left(\mathrm{B}_{s}\right)\right] d s=0 .
$$

Whenever this property holds only locally, which means for all products $\chi_{\Lambda} q \in$ $\mathcal{K}\left(\mathbb{P}^{D}\right)$ with characteristic functions $\chi_{\Lambda}$ of compact sets $\Lambda$ in $\mathcal{M}$, we write $q \in$ $\mathcal{K}_{\text {loc }}\left(\mathbb{P}^{D}\right)$. If a real-valued function $q$ satisfies $q^{+} \in \mathcal{K}_{\text {loc }}\left(\mathbb{P}^{D}\right)$ and $q^{-} \in \mathcal{K}\left(\mathbb{P}^{D}\right)$ then it is called Kato decomposable, symbolized as $q \in \mathcal{K}_{ \pm}\left(\mathbb{P}^{D}\right)$.

Remarks 33. If a function has the global or local Kato property for one choice of $D>0$, then this holds for any $D>0$. The reason to include $D$ in the definition is merely for the consistency of notation.

If the Ricci curvature of a Riemannian manifold is bounded from below, then the kernel is on compact sets up to any finite time uniformly bounded away from zero 45,46 . As a consequence, the local Kato property implies local integrability with respect to the volume measure, $\mathcal{K}_{l o c}\left(\mathbb{P}^{D}\right) \subset L_{l o c}^{1}(m)$.

The following lemma goes back to Khaśminskii [49]. Our discussion of the Kato class proceeds along the nice exposition in [50, Section 1.2]. The sole purpose of the following passage is to show that functions from the Kato class can be viewed as infinitesimally form-bounded perturbations of the Dirichlet and Bochner Laplacians.

Lemma 34. Suppose $0 \leq q \in \mathcal{K}\left(\mathbb{P}^{D}\right)$, and $t>0$ is chosen such that

$$
\kappa:=\sup _{x \in \mathcal{M}} \mathbb{E}_{x}^{D}\left[\int_{0}^{t} q\left(\mathrm{~B}_{r}\right) d r\right]<1,
$$

then

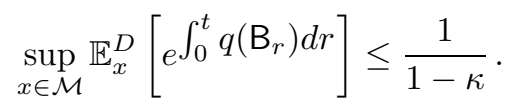


Proof. We refer to the proof in [50, Lemma 2.1], which is concerned with the special case of Brownian motion in Euclidean space. However, the formulation given there transfers literally without modification to the general situation on manifolds. The essential steps are to expand the exponential, to use time-ordering and the Markov property, and to inductively apply the assumption to obtain a geometric series in $\kappa$.

Consequence 35. Khaśminskii's lemma implies that for $q \in \mathcal{K}\left(\mathbb{P}^{D}\right)$, the mapping $Q_{t}$ given by

$$
Q_{t} \phi(x):=\mathbb{E}_{x}^{D}\left[e^{\left.\int_{0}^{t} q\left(\mathrm{~B}_{r}\right) d r_{\phi}\left(\mathrm{B}_{t}\right)\right]}\right.
$$

has a bound $\left\|Q_{t}\right\|_{\infty, \infty}:=\sup _{x \in \mathcal{M},\|\phi\|_{\infty}=1}\left|Q_{t} \phi(x)\right| \leq e^{C t} /(1-\kappa)$ on $L^{\infty}(m)$ with exponential growth in $t$. Thus $\left\{Q_{t}\right\}_{t \geq 0}$ is a semigroup of operators on $L^{\infty}(m)$.

Proof. Again, we refer to [50, Theorem 2.2]. The idea is to split $[0, t]$ into subintervals, to inductively use Lemma 34 in conjunction with the Markov property, and to bound the resulting expression with an exponential.

Lemma 36. Given a non-negative function $q \in \mathcal{K}\left(\mathbb{P}^{D}\right)$, then for any $c_{1}>0$ there is a $c_{2}>0$ such that

$$
\int_{\mathcal{M}} q|\phi|^{2} d m \leq c_{1} D \mathcal{E}(\phi, \phi)+c_{2}\|\phi\|_{2}^{2}
$$

whenever $\phi \in \mathcal{Q}(\mathcal{E})$. In other words, functions from the Kato class act as infinitesimally form-bounded perturbations of $-D \Delta$.

Proof. The proof proceeds in two steps.

Step 1. For $q \in \mathcal{K}\left(\mathbb{P}^{D}\right)$, the expression (49) defines a strongly continuous semigroup $\left\{Q_{t}\right\}_{t \geq 0}$ of bounded, self-adjoint operators $Q_{t}$ on $L^{2}(m)$.

The preceding lemma together with the Jensen and Cauchy-Schwarz inequalities establish the boundedness,

$$
\begin{aligned}
& \int_{\mathcal{M}}\left|\mathbb{E}_{x}^{D}\left[e^{\int_{0}^{t} q\left(\mathrm{~B}_{s}\right) d s} \phi\left(\mathrm{B}_{t}\right)\right]\right|^{2} d m(x) \\
& \leq \int_{\mathcal{M}} \mathbb{E}_{x}^{D}\left[e^{2 \int_{0}^{t} q\left(\mathrm{~B}_{s}\right) d s}\right] \mathbb{E}_{x}^{D}\left[\left|\phi\left(\mathrm{B}_{t}\right)\right|^{2}\right] d m(x) \leq \frac{1}{1-\kappa} e^{C t}\|\phi\|_{2}^{2} .
\end{aligned}
$$

Because of the Markovian semigroup property, it is enough to show strong continuity at $t=0$. To this end, we consider

$$
\begin{aligned}
& \lim _{t \searrow 0} \int_{\mathcal{M}}\left|\mathbb{E}_{x}^{D}\left[\left(e^{\int_{0}^{t} q\left(\mathrm{~B}_{s}\right) d s}-1\right) \phi\left(\mathrm{B}_{t}\right)\right]\right|^{2} d m(x) \\
& \leq \lim _{t \searrow 0} \int_{\mathcal{M}} \mathbb{E}_{x}^{D}\left[\left(e^{\int_{0}^{t} q\left(\mathrm{~B}_{s}\right) d s}-1\right)^{2}\right] \mathbb{E}_{x}^{D}\left[\left|\phi\left(\mathrm{B}_{t}\right)\right|^{2}\right] d m(x) \\
& \leq \lim _{t \searrow 0} \sup _{x \in \mathcal{M}} \mathbb{E}_{x}^{D}\left[e^{2 \int_{0}^{t}|q|\left(\mathrm{B}_{s}\right) d s}-1\right] \int \mathbb{E}_{x}^{D}\left[\left|\phi\left(\mathrm{B}_{t}\right)\right|^{2}\right] d m(x) .
\end{aligned}
$$

The last step involves Hölder's inequality and the elementary estimate $\left(e^{c}-1\right)^{2} \leq$ $e^{2|c|}-1$ for any real number $c \in \mathbb{R}$. Using the definition of the Kato class in this estimate shows that the limit of the $L^{2}$-norm in (53) vanishes. 
Moreover, by the time reversal invariance of Brownian motion each $Q_{t}$ is seen to be self-adjoint, and according to the Hille-Yosida theorem, there is a semibounded, self-adjoint generator of the semigroup. Given any $c_{1}>0$, we choose a suitable constant $c_{2}$ such that replacing $q$ by $\tilde{q}:=q / c_{1}-c_{2} / c_{1}$ in the above procedure yields a contraction semigroup.

Step 2. If we approximate $\tilde{q} \in \mathcal{K}\left(\mathbb{P}^{D}\right)$ with a sequence of semibounded functions $\tilde{q}_{l}:=\min \{\tilde{q}, l\}$, then for any $\phi \in \mathcal{Q}(\mathcal{E})$ the generator of the contraction semigroup $\widetilde{Q}_{t}$ associated with the function $\tilde{q}_{l}$ gives rise to a quadratic form

$$
\lim _{t \searrow 0} \frac{1}{t}\left(\phi, \widetilde{Q}_{t} \phi-\phi\right)=-D \mathcal{E}(\phi, \phi)-\frac{c_{2}}{c_{1}}\|\phi\|_{2}^{2}+\left(\phi, \min \left\{\frac{q}{c_{1}}, l+\frac{c_{2}}{c_{1}}\right\} \phi\right) \leq 0
$$

because $-D \Delta$ is essentially self-adjoint and the multiplication by $\tilde{q}_{l}$ is a bounded operator. The contractivity of the semigroup furnishes the last inequality, which in turn yields the form-boundedness condition (50) by monotone convergence in the limit $l \rightarrow \infty$.

Proposition 37. Let $\mathcal{L}$ be a Hermitian line bundle with a connection and an associated length-preserving horizontal transport $H$. Suppose the base manifold $\mathcal{M}$ is Riemannian and Brownian complete, equipped with a family of Brownian motion measures $\left\{\mathbb{P}_{x}^{D}\right\}_{x \in \mathcal{M}}$ having a common diffusion constant $D>0$. Then $q \in \mathcal{K}\left(\mathbb{P}^{D}\right)$ is also a form-bounded perturbation of the negative Bochner Laplacian $-\Delta^{\mathcal{L}}$.

Proof. To make contact with the preceding lemma, we fix $\psi \in L^{2}(h m)$ and define a function $\phi \in L^{2}(m)$ with values $\phi(x):=\sqrt{h_{x}(\psi(x), \psi(x))}$.

We may now verify the $L^{2}$-boundedness of the operators $Q_{t}^{\mathcal{L}}$ given by

$$
Q_{t}^{\mathcal{L}} \psi(x):=\mathbb{E}_{x}^{D}\left[e^{\int_{0}^{t} q\left(\mathrm{~B}_{r}\right) d r} H_{\mathrm{B}, t}^{-1} \psi\left(\mathrm{B}_{t}\right)\right]
$$

with an estimate using that horizontal transport preserves the Hermitian metric and the same strategy as in the preceding lemma,

$$
\begin{aligned}
\sqrt{h_{x}\left(Q_{t}^{\mathcal{L}} \psi(x), Q_{t}^{\mathcal{L}} \psi(x)\right)} & \leq \mathbb{E}_{x}^{D}\left[e^{\int_{0}^{t} q\left(\mathrm{~B}_{r}\right) d r} \sqrt{h_{x}\left(H_{\mathrm{B}, t}^{-1} \psi\left(\mathrm{B}_{t}\right), H_{\mathrm{B}, t}^{-1} \psi\left(\mathrm{B}_{t}\right)\right)}\right] \\
& =\mathbb{E}_{x}^{D}\left[e^{\int_{0}^{t} q\left(\mathrm{~B}_{r}\right) d r} \sqrt{h_{\mathrm{B}_{t}}\left(\psi\left(\mathrm{B}_{t}\right), \psi\left(\mathrm{B}_{t}\right)\right)}\right] \\
& =\mathbb{E}_{x}^{D}\left[e^{\int_{0}^{t} q\left(\mathrm{~B}_{r}\right) d r} \phi\left(\mathrm{B}_{t}\right)\right]=e^{-t S_{D, q} \phi(x) .}
\end{aligned}
$$

A similar estimate gives

$$
\left(\psi, Q_{t}^{\mathcal{L}} \psi-\psi\right) \leq\left(\phi, Q_{t} \phi-\phi\right)
$$

and thus together with the preceding lemma the desired form-boundedness.

Consequence 38. Therefore, any $q \in \mathcal{K}\left(\mathbb{P}^{D}\right)$ may be used to define a formbounded perturbation of $-D \Delta$ or $-D \Delta^{\mathcal{L}}$ in order to define a self-adjoint Schrödinger operator. One may also use $\mathfrak{S}_{D, q^{+}}$or $\mathfrak{S}_{D, q^{+}}^{\mathcal{L}}$ as the unperturbed forms and thus extend this construction to define a Schrödinger operator $\mathfrak{S}_{D, q}$ with $q \in \mathcal{K}_{ \pm}\left(\mathbb{P}^{D}\right)$. 
Proposition (Feynman-Kac formula) 39. Let $\mathcal{L}$ be a Hermitian line bundle with a connection and a length-preserving horizontal transport $H$. Suppose the base manifold $\mathcal{M}$ is Riemannian and Brownian complete. Denote by $m$ the natural volume measure on $\mathcal{M}$ and by $\mathbb{P}^{D}$ a family of Brownian-motion measures having a common diffusion constant $D>0$.

If $q \in \mathcal{K}_{ \pm}\left(\mathbb{P}^{D}\right)$, then the image of a section $\psi \in L^{2}(h m)$ under the semigroup $e^{-t S_{D, q}^{\mathcal{L}} \text { generated by the self-adjoint Schrödinger operator } S_{D, q}^{\mathcal{L}} \text { has the probabilistic }}$ representation

$$
e^{-t S_{D, q}^{\mathcal{L}} \psi(x)}=\mathbb{E}_{x}^{D}\left[e^{-\int_{0}^{t} q\left(\mathrm{~B}_{r}\right) d r} H_{\mathrm{B}, t}^{-1} \psi\left(\mathrm{B}_{t}\right)\right] .
$$

The inverse of the stochastic horizontal transport appearing in this equation can either be understood by appealing to localized expressions [51], i.e. one restricts to a subspace of the probability space by introducing exit times of local coordinate patches and then reformulates the horizontal transport in a local trivialization, or one interprets (62) as a shorthand for

$$
h_{x}\left(u, e^{-t S_{D, q}^{\mathcal{L}}} \psi(x)\right)=\mathbb{E}_{x}^{D}\left[e^{-\int_{0}^{t} q\left(\mathrm{~B}_{r}\right) d r} h_{\mathrm{B}_{t}}\left(H_{\mathrm{B}, t} u, \psi\left(\mathrm{B}_{t}\right)\right)\right]
$$

with an arbitrary reference vector $u \in \mathcal{L}_{x}$.

Proof. The proof of formula (62) is given in Appendix C.

Consequence 40. Let $\mathcal{L}$ be a holomorphic Hermitian line bundle, assume its base manifold $\mathcal{M}$ is Kähler, and let $g$ denote the real part of the Kähler metric. Let $\left\{Z_{k}\right\}_{k=1}^{d / 2}$ denote a local holomorphic orthonormal frame of $T^{(1,0)} \mathcal{M}$. If the curvature term $\rho=\sum_{k=1}^{d / 2} R_{\bar{Z}_{j}, Z_{j}}$ determined by the connection of the line bundle $\mathcal{L}$ is Kato decomposable, then $-\Delta^{\mathcal{L}}$ and $-\Delta^{(0, \bullet)}$ have the same domain and are essentially self-adjoint on $C_{c \mathcal{L}}^{\infty}(\mathcal{M})$. If $f$ is also Kato decomposable, then the Feynman-Kac formula (62) with $q=D \rho+f$ gives an expression for the Schwartz kernel of the semigroup generated by $S_{2 D, f}^{(0, \bullet)}$.

Definition 41. With the help of the distance function on $\mathcal{M}$, the space $C_{\mathcal{M}}([0, t])$ of continuous paths in $\mathcal{M}$ parametrized by an interval $[0, t]$ can be turned into a complete, separable metric space. In this setting, one may construct a regular conditional probability measure of $\mathbb{P}_{x}^{D}$ given $\mathrm{B}_{t}$ [52, Theorem 5.3.19]. We denote by $\mathbb{P}_{x, y}^{D, t}$ the Brownian bridge measure, that is the probability measure of the Brownian motion which starts at $\mathrm{B}_{0}=x$ and is conditioned to arrive at $\mathrm{B}_{t}=y$. It is understood as a regular conditional probability distribution of $\mathbb{P}_{x}^{D}$ given $\mathrm{B}_{t}$ [53, pp. 146-150].

Consequence 42. With the assumptions of Consequence 40, the Feynman-Kac formula (62) can be modified to give an expression for the integral kernel of the Schrödinger semigroup

$$
e^{-t S_{2 D, f}^{(0, \bullet)}(x, y)}=p_{D, t}(x, y) \mathbb{E}_{x, y}^{D, t}\left[e^{-\int_{0}^{t}\left(D \rho\left(\mathrm{B}_{r}\right)+f\left(\mathrm{~B}_{r}\right)\right) d r} H_{\mathrm{B}, t}^{-1}\right]
$$

generated by $S_{2 D, f}^{(0, \bullet)}$. Hereby, we use the unique heat kernel $\left\{p_{D, t}\right\}_{t>0}$ of $D \Delta$, the expectation with respect to the Brownian bridge measure $\mathbb{P}_{x, y}^{D, t}$ given above, and the inverse of the stochastic horizontal transport is understood as a linear mapping from $\mathcal{L}_{y}$ to $\mathcal{L}_{x}$. 
Definition 43. In the following results, we always consider a fixed Kato-decomposable symbol $f \in \mathcal{K}_{ \pm}\left(\mathbb{P}^{D}\right)$. To simplify notation, we choose a reference diffusion constant $D_{0}>0$ and abbreviate for $c \geq 0, v \in \mathcal{L}$ the section

$$
\eta_{c, v}:=e^{-S_{2 D_{0}, c f}^{(0, \bullet)}(\cdot, \pi(v)) v}
$$

obtained by keeping one end of the Schwartz kernel fixed.

Lemma 44. For any $v \in \mathcal{L}$ and $c \geq 0$, the section $\eta_{c, v}$ is contained in $L^{2}(h m)$. In addition, the mapping $c \mapsto \eta_{c, v}$ is strongly continuous.

Proof. Due to the linearity in $v$, it is enough to consider a vector of length $\|v\|=1$. The $L^{2}$-norm of $\eta_{c, v}$ can then be estimated by repeatedly using the Cauchy-Schwarz and Hölder inequalities:

$$
\begin{aligned}
\left\|\eta_{c, v}\right\|_{2} & \leq \sup _{\substack{v \in \mathcal{L}_{y},\|v\|=1 \\
\psi \in L^{2}(h m),\|\psi\|_{2}=1}}\left|\left(\eta_{c, v}, \psi\right)\right| \leq \sup _{\substack{v \in \mathcal{L},\|v\|=1 \\
\psi \in L^{2}(h m),\|\psi\|_{2}=1}}\left|h_{\pi(v)}\left(e^{-S_{2 D_{0}, c f}^{(0, \bullet)}} \psi(\pi(v)), v\right)\right| \\
& \leq \sup _{\|\psi\|_{2}=1, x \in \mathcal{M}}\left\|\mathbb{E}_{x}^{D_{0}}\left[e^{-\int_{0}^{1}\left(D_{0} \rho+c f\right)\left(\mathrm{B}_{t}\right) d t} H_{\mathrm{B}, 1}^{-1} \psi\left(\mathrm{B}_{1}\right)\right]\right\| \\
& \leq \sup _{\|\psi\|_{2}=1, x \in \mathcal{M}}\left(\mathbb{E}_{x}^{D_{0}}\left[e^{-2 \int_{0}^{1}\left(D_{0} \rho+c f\right)\left(\mathrm{B}_{t}\right) d t}\right] \mathbb{E}_{x}^{D_{0}}\left[\left\|\psi\left(\mathrm{B}_{t}\right)\right\|^{2}\right]\right)^{1 / 2} \\
& \leq\left(\left\|e^{-S_{D_{0}, 2 D_{0} \rho+2 c f}^{\mathcal{L}}}\right\|_{\infty, \infty}\right)^{1 / 2}\left\|p_{1}(\cdot, x)\right\|_{\infty}<\infty
\end{aligned}
$$

The finiteness results from the Kato decomposability of $\rho$ and $f$ and from the boundedness of the heat kernel $p_{1}$ [45, 46, 54].

To see the strong continuity of $\eta_{c, v}$ in $c$, we may for cimplicity assume $v$ to be again a normalized vector and consider two non-negative coupling constants $c$ and $c^{\prime}$. If $\psi \in L^{2}(h m)$ also has the $L^{2}$-norm $\|\psi\|_{2}=1$, then we may estimate

$$
\begin{gathered}
\left|\left(\eta_{c, v}-\eta_{c^{\prime}, v}, \psi\right)\right|=\mid \mathbb{E}_{\pi(v)}^{D_{0}}\left[\left(e^{-\int_{0}^{1}\left(D_{0} \rho+c f\right)\left(\mathrm{B}_{t}\right) d t}\right.\right. \\
\left.\left.-e^{-\int_{0}^{1}\left(D_{0} \rho+c^{\prime} f\right)\left(\mathrm{B}_{t}\right) d t}\right) h_{\mathrm{B}_{1}}\left(H_{\mathrm{B}, 1} v, \psi\left(\mathrm{B}_{1}\right)\right)\right] \mid \\
\leq\left(\mathbb{E}_{\pi(v)}^{D_{0}}\left[\left|h_{\mathrm{B}_{1}}\left(v, \psi\left(\mathrm{B}_{1}\right)\right)\right|^{2}\right]\right)^{1 / 2}\left(\mathbb { E } _ { \pi ( v ) } ^ { D _ { 0 } } \left[\left(e^{-\int_{0}^{1}\left(D_{0} \rho+c f\right)\left(\mathrm{B}_{t}\right) d t}\right.\right.\right. \\
\left.\left.\left.-e^{-\int_{0}^{1}\left(D_{0} \rho+c^{\prime} f\right)\left(\mathrm{B}_{t}\right) d t}\right)^{2}\right]\right)^{1 / 2} \\
\leq\left(\mathbb{E}_{\pi(v)}^{D_{0}}\left[h_{\mathrm{B}_{1}}\left(\psi\left(\mathrm{B}_{1}\right), \psi\left(\mathrm{B}_{1}\right)\right)\right]\right)^{1 / 2}\left(\mathbb { E } _ { \pi ( v ) } ^ { D _ { 0 } } \left[\left(e^{-\int_{0}^{1}\left(D_{0} \rho+c f\right)\left(\mathrm{B}_{t}\right) d t}\right.\right.\right. \\
\left.\left.\left.-e^{-\int_{0}^{1}\left(D_{0} \rho+c^{\prime} f\right)\left(\mathrm{B}_{t}\right) d t}\right)^{2}\right]\right)^{1 / 2} .
\end{gathered}
$$


Taking the supremum over $\psi,\|\psi\|_{2}=1$ on both sides together with the $L^{2}$-contraction property of the unperturbed heat semigroup generated by $D_{0} \Delta^{\mathcal{L}}$ yields

$$
\begin{aligned}
& \left\|\eta_{c, v}-\eta_{c^{\prime}, v}\right\|_{2}=\sup _{\psi \in L^{2}(h m),\|\psi\|_{2}=1}\left|\left(\eta_{c, v}-\eta_{c^{\prime}, v}, \psi\right)\right| \\
& \leq\left(\mathbb{E}_{\pi(v)}^{D_{0}}\left[\left(e^{-\int_{0}^{1}\left(D_{0} \rho+c f\right)\left(\mathrm{B}_{t}\right) d t}-e^{-\int_{0}^{1}\left(D_{0} \rho+c^{\prime} f\right)\left(\mathrm{B}_{t}\right) d t}\right)^{2}\right]\right)^{1 / 2} \\
& \leq 2\left(\mathbb{E}_{\pi(v)}^{D_{0}}\left[e^{2 \int_{0}^{1}\left(D_{0} \rho^{-}+c_{0} f^{-}\right)\left(\mathrm{B}_{t}\right) d t}\right]\right)^{1 / 2} .
\end{aligned}
$$

The purpose of the last estimate is to show that with the help of some large $c_{0}$, dominated convergence applies to (72) in the limit $c^{\prime} \rightarrow c$.

Theorem (Daubechies-Klauder formula) 45. Let $\mathcal{L}$ be a holomorphic line bundle with a Hermitian metric $h$, suppose its base manifold $\mathcal{M}$ is equipped with a Kähler metric, denote its real part by $g$ and the natural volume measure by $m$. Let $\mathcal{M}$ be Riemannian complete with Ricci curvature bounded below, to ensure Brownian completeness. Let the real-valued function $f: \mathcal{M} \rightarrow \mathbb{R}$ be Kato decomposable with respect to the Brownian-motion measure $\mathbb{P}^{D}$ on $\mathcal{M}$, where the diffusion constant $D>0$ is arbitrary. In addition, suppose the curvature term $\rho$ defined in Proposition 20 is also Kato decomposable. To include the case when $T_{f}$ is not densely defined, we denote by $K_{f}$ the orthogonal projector onto the closure of the form domain $\mathcal{Q}\left(\mathcal{T}_{f}\right)$ in $L_{\text {hol }}^{2}(\mathrm{hm})$.

With these assumptions the integral kernel of the Berezin-Toeplitz semigroup $\left\{e^{-t T_{f}} K_{f}\right\}_{t \geq 0}$ is for $t>0$ given by the probabilistic expression

$$
\left(e^{-t T_{f}} K_{f}\right)(x, y)=\lim _{D \rightarrow \infty} p_{D, t}(x, y) \mathbb{E}_{x, y}^{D, t}\left[e^{-\int_{0}^{t}\left(D \rho\left(\mathrm{B}_{r}\right)+f\left(\mathrm{~B}_{r}\right)\right) d r} H_{\mathrm{B}, t}^{-1}\right] .
$$

In particular, we obtain the reproducing kernel $K$ of $L_{h o l}^{2}(h m)$ as a special case of this formula when $f=0$.

Proof. In conjunction with the specific use of the Feynman-Kac formula in Consequence 42, it is equivalent to show that the integral kernel of the Berezin-Toeplitz semigroup $e^{-t T_{f}} K_{f}$ on $L_{h o l}^{2}(h m)$ is for $t>0$ given by the pointwise limit

$$
\left(e^{-t T_{f}} K_{f}\right)(x, y)=\lim _{D \rightarrow \infty} e^{-t S_{D, f}^{(0, \bullet)}}(x, y),
$$

where $S_{D, f}^{(0, \bullet)}$ is the semibounded Schrödinger operator defined by equation (41).

We borrow the strategy of [55] and accommodate it to the manifold situation and the case of unbounded $f$. The key to the present generalization is the use of monotone form convergence.

We have to show that for $u, v \in \mathcal{L}$ with base points $x, y \in \mathcal{M}$ the equation

$$
\lim _{D \rightarrow \infty} h_{x}\left(u, e^{-t S_{D, f}^{(0, \bullet)}}(x, y) v\right)=\left(e_{u}, e^{-t T_{f}} K_{f} e_{v}\right)
$$

holds, which by Consequence 9 characterizes the continuous integral kernel of $e^{-t T_{f}} K_{f}$ on $L_{h o l}^{2}(h m) \subset L^{2}(h m)$.

To see (76), we use the semigroup property and express the integral kernel with some choice of $D_{0}>0$ in a scalar product

$$
h_{x}\left(u, e^{-t S_{D, f}^{(0, \bullet)}}(x, y) v\right)=\left(\eta_{D_{0} / D, u}, \exp \left(-t S_{D-2 D_{0},\left(D-2 D_{0}\right) f / D}^{(0, \bullet)}\right) \eta_{D_{0} / D, v}\right)
$$


that converges in the limit $D \rightarrow \infty$ to

$$
\lim _{D \rightarrow \infty} h_{x}\left(u, e^{-t S_{D, f}^{(0, \bullet)}}(x, y) v\right)=\left(\eta_{0, u}, e^{-t T_{f}} K_{f} \eta_{0, v}\right) .
$$

This can be deduced from the strong continuity of $\eta_{c, w}$ in $c$ for any $w \in \mathcal{L}$ and the strong convergence stated in Consequence 31 together with the uniform boundedness (according to the Banach-Steinhaus theorem) of $\exp \left(-t S_{D-2 D_{0},\left(D-2 D_{0}\right) f / D}^{(0, \bullet)}\right.$ in $D>2 D_{0}$.

To finish the proof, we observe that the right-hand side of (78) is an integral kernel for $\exp \left(-t T_{f}\right) K_{f}$ on $L_{h o l}^{2}(h m)$ that is, in addition, continuous in $x$ and $y$ and therefore coincides with the right-hand side of (76). The continuity of (78) is guaranteed by the continuity of the heat kernel derived in Appendix B, and with $K_{f} \exp \left(-S_{D_{0}, 0}^{(0, \bullet)}\right)=K_{f}$ it can be checked that it indeed constitutes an integral kernel.

Remarks 46. The probabilistic representation of Berezin-Toeplitz semigroups according to formula (74) has also been called a Wiener-regularized path integral, because it gives meaning to similar, non-rigorous versions of such path integrals.

With particular choices of holomorphic line bundles over homogeneous Kähler manifolds related to Lie-group representations [56], formula (74) yields an analogue of the situations considered by Daubechies, Klauder, and Paul 20,24. The particular Lie groups in consideration are the Heisenberg-Weyl group, $S U(2)$ and the affine group. In each of these cases, the complex dimension of the manifold $\mathcal{M}$ is $n=1$, the Riemannian metric $g$ is the real part of the Kähler metric, and the imaginary, skew-symmetric part is in the prequantum relation (33) with the curvature of the line bundle. For an explicit result that does not satisfy this relation, see the treatment in [55] or [57. The result given there differs from that of Daubechies and Klauder [20] by a conformal rescaling of the Kähler metric on the base manifold.

It is worth pointing out that with a suitable analyticity argument, one could obtain from formula (75) the probabilistic expression for the Schwartz kernel of the unitary group $e^{-i t \bar{T}_{f}}$, which was a primary motivation for 1924 . The case of bounded $f$ may be treated according to [55]. The techniques in \$58 appear suitable for a generalization to $f \in \mathcal{K}\left(\mathbb{P}^{D}\right)$, but the Kato decomposable case seems to require an additional effort.

\section{Summary AND Outlook}

In this work, we have studied a coordinate-independent quantization prescription in the spirit of Berezin and its representation by Wiener-regularized path integrals according to an idea of Daubechies and Klauder. In the present version, these path integrals express semigroups that are generated by self-adjoint semibounded Berezin-Toeplitz operators on a generalized Bergman space.

The first results concerned conditions that guarantee self-adjointness and semiboundedness of Berezin-Toeplitz operators. The use of quadratic forms provided a convenient framework to develop such conditions, which in the course of Sections 2 to 4 evolved from rather abstract form-boundedness to the more concrete requirement in terms of the Kato class. The Dirichlet Laplacian provided a natural geometric characterization of this class. Besides the Kato class, the holomorphic Laplacian proved central to our implementation of the concept by Daubechies and Klauder on Kähler manifolds. More specifically, we considered perturbations of the 
holomorphic Laplacian in conjunction with a limiting procedure and the FeynmanKac formula to construct Wiener-regularized path integrals, a probabilistic representation of the Schwartz kernel for the semigroup generated by a Berezin-Toeplitz operator. One implication of this construction was that the reproducing kernel of a space of holomorphic, square-integrable sections in a holomorphic Hermitian line bundle over a Kähler manifold could be expressed in purely geometric terms.

The fundamental idea behind all those results was the relation between BerezinToeplitz operators and Schrödinger operators, which enabled us to transfer all the relevant analytic and probabilistic techniques.

As to further developments, one may ask whether Wiener-regularized path integrals can also be found for continuous representations without underlying complex structures. A step in this direction this has been pointed out by [25] with the use of Dirac operators and $\operatorname{spin}^{c}$ structures. Indeed, the completeness argument for the Hilbert space in Appendix A could be applied to a space of merely harmonic functions, since all that is required are mean-value and continuity properties. In addition, the context of Dirac operators may provide enough analytic tools to replace techniques that so far relied on the presence of complex structures. Another ramification is the concept of path transformations, well-known in the study of Schrödinger operators [58]. Indeed, one may use an invariance property of Brownian motion under harmonic morphisms to relate the resolvents of different BerezinToeplitz operators to one another [59. Finally, it may be worthwhile to study the use of Wiener-regularized path integrals to extend the correspondence principle from the compact Kähler case to non-compact manifolds. Probabilistic representations have often been useful to bridge between different function spaces. In this case, a suitable procedure of approximating non-compact Kähler manifolds by compact ones in the path-integral representation could help enlarging the validity of the correspondence principle.

Acknowledgements. Heartfelt thanks go to John Klauder whose strong intuition proved invaluable in our discussions of geometric principles in quantization procedures.

\section{Appendix A. Completeness of the Generalized Bergman Space}

In this part of the appendix, we show that the spaces of holomorphic, squareintegrable sections $L_{h o l}^{2}(\mathrm{hm})$ we consider are indeed Hilbert spaces. The main part is a localization argument that reduces the setting to that of the space of squareintegrable holomorphic functions on the unit ball as studied by Bergman [60].

Let $d^{2 n} z$ denote the Lebesgue measure on $\mathbb{C}^{n}$. We recall that the open ball $B(x, r):=\left\{y \in \mathbb{C}^{n}: \sum_{l=1}^{n}\left|y_{l}-x_{l}\right|^{2}<r^{2}\right\}$ centered at $x \in \mathbb{C}^{n}$ with radius $r \geq 0$ has the volume $\int_{\mathbb{C}^{n}} \chi_{B(x, r)}(z) d^{2 n} z=\pi^{n} r^{2 n} / n$ !, where $n !:=1 \cdot 2 \cdots n$ denotes the factorial of $n$.

Lemma A.1. The inner-product space $L_{h o l}^{2}(B(0,1))$ of holomorphic functions that are square-integrable with respect to the Lebesgue measure on $B(0,1)$ is complete in the norm-topology induced by the usual $L^{2}$-inner product.

Proof. Let $\left(f_{j}\right)_{j \in \mathbb{N}}$ be a Cauchy sequence in $L_{h o l}^{2}(B(0,1))$. First we show uniform convergence on all compact sets $C$ inside $B(0,1)$. For any such set $C$, we can find a nonzero safety radius $r>0$ smaller than the distance from $C$ to the boundary of $B(0,1)$. Using the mean value property for holomorphic functions, we can express the difference of two function values at a point $x \in C$ as the difference between the 
averages of the two functions, each computed for a disk centered at $x$. Then Jensen's inequality in conjunction with the convexity of the square-modulus function $c \mapsto|c|^{2}$ on $\mathbb{C}$ yields

$$
\begin{aligned}
& \sup _{x \in C}\left|f_{j}(x)-f_{k}(x)\right|^{2} \leq\left(\frac{n !}{\pi^{n} r^{2 n}}\right)^{2} \sup _{x \in C} \int_{B(x, r)}\left|f_{j}(z)-f_{k}(z)\right|^{2} d^{2 n} z \\
\leq & \left(\frac{n !}{\pi^{n} r^{2 n}}\right)^{2} \int_{B(0,1)}\left|f_{j}(z)-f_{k}(z)\right|^{2} d^{2 n} z=\left(\frac{n !}{\pi^{n} r^{2 n}}\right)^{2}\left\|f_{j}-f_{k}\right\|_{2}^{2} .
\end{aligned}
$$

The right-hand side can be made arbitrarily small and thus the sequence $\left(f_{j}\right)_{j \in \mathbb{N}}$ converges uniformly on $C$. By a standard argument in complex analysis, we conclude that the pointwise limit defines a holomorphic function $f: f(z)=\lim _{j \rightarrow \infty} f_{j}(z)$ in $B(0,1)$.

That the convergence $f_{j} \rightarrow f$ is also in the sense of the $L^{2}$-norm follows from the Cauchy property of the sequence and from $\left\|f-f_{k}\right\|_{2} \leq \liminf \lim _{j \rightarrow \infty}\left\|f_{j}-f_{k}\right\|_{2}$ due to pointwise convergence and Fatou's lemma.

Theorem A.2. Let $\mathcal{L}$ be a holomorphic line bundle with the complex, $n$-dimensional base manifold $\mathcal{M}$. Suppose the fibers of $\mathcal{L}$ are equipped with a Hermitian metric $h$ and $M$ possesses a volume form $m$, both forms non-degenerate and smooth. Then the space $L_{h o l}^{2}(h m)$ of holomorphic, square-integrable sections in $\mathcal{L}$ forms a Hilbert space.

Proof. To begin with, we choose an atlas of local trivializations $\left\{\xi_{j}\right\}_{j \in I}$ and corresponding reference sections $s_{j}$. That is, in each chart domain $U_{j}$ of the underlying atlas covering $\mathcal{M}$ we choose $s_{j}$ such that the composition $\xi_{j} \circ s_{j}$ has the constant value one in the second component. Choosing $\xi_{j}: \pi^{-1}\left(U_{j}\right) \rightarrow V_{j} \times \mathbb{C}$ determines $s_{j}$. Thus, we can identify each section $\psi$ with a set of functions $\left\{\psi_{j}: U_{j} \rightarrow \mathbb{C}\right\}_{j \in I}$ that satisfy $\left.\psi\right|_{U_{j}}=\psi_{j} s_{j}$ via multiplication in the fibers.

Similarly as in the preceding completeness proof, it is enough to show that any given Cauchy sequence converges pointwise to a holomorphic section, which is a holomorphic representative of the limit in the $L^{2}$-sense.

To this end, we note that with the local reference sections, the Cauchy sequence $\left\{\psi^{(l)}\right\}_{l \in \mathbb{N}}$ is represented by a sequence of holomorphic functions $\psi_{j}^{(l)}: U_{j} \rightarrow \mathbb{C}$. The image measure of $m$ under a chart $\phi_{j}: U_{j} \rightarrow V_{j} \subset \mathbb{C}^{n}$ has a density with respect to the Lebesgue measure on $V_{j}, d m(z)=m_{j}(z) d^{2 n} z$.

Suppose we have chosen local trivializations $\xi_{j}$ with the range of each underlying chart $\phi_{j}$ being a ball of radius $r_{j}$ centered at the origin. The non-degeneracy and smoothness of $h$ and $\mu$ imply that for each $U_{j}$, there is a strictly positive lower bound $0<\epsilon_{j}<m_{j}(z)$. We deduce that $\left\{\psi_{j}^{(l)}\right\}_{l \in \mathbb{N}}$ is a Cauchy sequence of holomorphic functions in the conventional Bergman space $L_{h o l}^{2}\left(V_{j}, \epsilon_{j} d^{2 n} z\right)$. According to the preceding lemma, the sequence converges pointwise to a holomorphic function. The limits obtained on each $V_{j}$ can then be recombined with the help of the reference sections $s_{j}$ to give a global, holomorphic section. This limit section is the holomorphic representative that coincides almost everywhere with the limit of the Cauchy sequence $\left\{\psi^{(l)}\right\}_{l \in \mathbb{N}}$ taken in $L^{2}(h m)$. 


\section{Appendix B. Smoothness of Heat Kernels}

The crucial idea used in the construction of the heat kernel is that the index $m \in$ $\mathbb{N}$ of a Sobolev space $W^{m, 2}\left(\mathbb{R}^{d}\right)$ controls the regularity properties of its functions.

To simplify the notation, we introduce a customary $d$-dimensional multi-index $j=\left(j_{1}, j_{2}, \ldots j_{d}\right)$ with non-negative components $j_{1}, j_{2}, \ldots j_{d} \in \mathbb{Z}^{+}$and define its degree by $|j|:=\sum_{k=1}^{d} j_{k}$. For $k=\left(k_{1}, k_{2}, \ldots, k_{d}\right) \in \mathbb{R}^{d}$, we abbreviate $k^{j}:=$ $k_{1}^{j_{1}} k_{2}^{j_{2}} \ldots k_{d}^{j_{d}}$.

Definition B.1. The Sobolev space $W^{m, 2}\left(\mathbb{R}^{d}\right)$ with $m \in \mathbb{N}$ consists of square-integrable functions $f: \mathbb{R}^{d} \rightarrow \mathbb{C}$ having Fourier transforms $\tilde{f}: k \mapsto \int_{\mathbb{R}^{d}} e^{-i k \cdot x} f(x) d^{d} x$ that render the Sobolev norm $\int_{\mathbb{R}^{d}}|\tilde{f}(k)|^{2}\left(1+k^{2}\right)^{m} d^{d} k$ finite. Equipped with this norm, $W^{m, 2}\left(\mathbb{R}^{d}\right)$ is complete.

Lemma B.2. Given a fixed maximal degree $0 \leq l<m-d / 2$, the linear functionals

$$
\delta_{x}^{(j)}: f \longmapsto \int_{\mathbb{R}^{d}} k^{j} e^{i k \cdot x} \tilde{f}(k) \frac{d^{d} k}{(2 \pi)^{d}}
$$

with $x \in \mathbb{R}^{d}$ and $|j| \leq l$ are uniformly bounded on $W^{m, 2}\left(\mathbb{R}^{d}\right)$. Moreover, in this case any function $f \in W^{m, 2}\left(\mathbb{R}^{d}\right)$ has an $l$-times continuously differentiable representative $x \mapsto \delta_{x}^{(0)}(f)$.

Proof. This statement is a rearrangement of [61, Theorem 12.29].

Proposition B.3. Given a complex line bundle $\mathcal{L}$ with a Riemannian base manifold $\mathcal{M}$, the semigroup generated by the self-adjoint Bochner-Laplacian $-\Delta^{\mathcal{L}}$ as defined in (21) has a Schwartz kernel $\left\{p_{t}^{\mathcal{L}}(x, y): \mathcal{L}_{y} \rightarrow \mathcal{L}_{x}\right\}_{t>0 ; x, y \in \mathcal{M}}$ that is smooth in the parameters $t, x$ and $y$.

Proof. As a first step, we establish properties of point-evaluation functionals on Sobolev-type spaces of sections in $\mathcal{L}$.

A section $\sigma=\sigma_{j} s_{j}$ with compact support in the domain of a chart $\phi_{j}: U_{j} \rightarrow$ $V_{j} \subset \mathbb{R}^{d}$ can be identified with $\sigma_{j} \circ \phi_{j}^{-1}$, and because of its compact support canonically extends by zero on the remaining part of $\mathbb{R}^{d}$. Due to the smoothness and non-degeneracy of the metric, its eigenvalues obtain a maximum and a nonzero minimum on the support of $\sigma$. Therefore, $\Delta^{\mathcal{L}}$ acts locally as a uniformly elliptic operator and allows estimating $\left(\sigma,\left(1-\Delta^{\mathcal{L}}\right) \sigma\right)$ from above and below by multiples of the Sobolev-norm of the function $\sigma_{j}$ in $W^{1,2}\left(\mathbb{R}^{d}\right)$. By an inductive procedure, the same technique gives estimates for $\left(\sigma,\left(1-\Delta^{\mathcal{L}}\right)^{m} \sigma\right)$ in terms of norms in $W^{m, 2}\left(\mathbb{R}^{d}\right)$. From now on, we refer to the Sobolev-type space of sections $\psi$ having the finite norm $\|\psi\|\|:=\|\left(1-\Delta^{\mathcal{L}}\right)^{m / 2} \psi \|$ as $\mathcal{W}_{\mathcal{L}}^{m, 2}(\mathcal{M})$.

In analogy to the Sobolev spaces on $\mathbb{R}^{d}$, the linear functional $\vartheta_{u}: \psi \mapsto h_{x}(u, \psi(x))$ evaluating sections at $x=\pi(u)$ is for sufficiently large $m$ bounded in $\mathcal{W}_{\mathcal{L}}^{m, 2}(\mathcal{M})$. At first, the bound is only valid on the closed subspace of sections $\sigma$ with support in a sufficiently small compact set $C$ containing $x$. However, the sections in the orthogonal complement of the subspace vanish on $C$ and thus the bound of $\vartheta_{u}$ passes unchanged to the whole of $\mathcal{W}_{\mathcal{L}}^{m, 2}(\mathcal{M})$ 62. By a similar localization argument and the preceding lemma, $u \mapsto \vartheta_{u}$ is seen to be smooth, and so are all the sections $\psi$ in $\mathcal{W}_{\mathcal{L}}^{m, 2}(\mathcal{M})$. 
The next step of the proof makes use of these smoothness properties to construct the heat kernel.

In the spectral representation we see that for fixed $m \in \mathbb{N}$ and $t_{0}>0$, the operators $\left(1-\Delta^{\mathcal{L}}\right)^{m / 2} e^{t \Delta^{\mathcal{L}}}$ are uniformly in $t \geq t_{0}$ bounded on $L^{2}(h \mu)$. In consequence, the semigroup $e^{t \Delta^{\mathcal{L}}}$ is bounded as a mapping from $L^{2}(h \mu)$ into all Sobolev-type spaces $\mathcal{W}^{m, 2}(\mathcal{M})$, and choosing a sufficiently large $m$ proves that the functional $\psi \mapsto \vartheta_{u}\left(e^{t \Delta^{\mathcal{L}}} \psi\right)$ is bounded and linear in $\psi \in L^{2}(h m)$. By the Riesz Representation Theorem and due to the linearity of $u \mapsto \vartheta_{u}$ in the fibers, there is a vector $q_{t}(\cdot, \pi(u)) u$ in $L^{2}(h m)$ such that $\vartheta_{u}\left(e^{t \Delta^{\mathcal{L}}} \psi\right)=\left(q_{t}(\cdot, \pi(u)) u, \psi\right)$ for all $\psi \in L^{2}(h m)$. By the smoothness of $u \mapsto\left(q_{t}(\cdot, \pi(u)) u, \psi\right)$ and a uniform boundedness argument, the map $u \mapsto q_{t}(\cdot, \pi(u)) u$ is smooth in the strong sense.

In addition, the map $t \mapsto q_{t}(\cdot, \pi(u)) u$ is also smooth, because $\left(1-\Delta^{\mathcal{L}}\right)^{m / 2} e^{t \Delta^{\mathcal{L}}}$ is real analytic in $t>0$.

In the last step, we define a smooth kernel $p_{t}^{\mathcal{L}}(x, y)$ by

$$
h_{x}\left(u, p_{t}^{\mathcal{L}}(\pi(u), \pi(v)) v\right):=\left(q_{t / 2}(\cdot, \pi(u)) u, q_{t / 2}(\cdot, \pi(v)) v\right)
$$

and claim that it is a Schwartz kernel for $e^{t \Delta^{\mathcal{L}}}$. Using the definition (82), the equation

$$
\int_{\mathcal{M} \times \mathcal{M}} h_{x}\left(\psi(x), p_{t}(x, y) \sigma(y)\right) d \mu(x) d \mu(y)=\left(e^{-t \Delta^{\mathcal{L}} / 2} \psi, e^{-t \Delta^{\mathcal{L}} / 2} \sigma\right)
$$

follows for sections $\psi, \sigma \in C_{c \mathcal{L}}^{\infty}(\mathcal{M})$. The self-adjointness and boundedness of $e^{-t \Delta^{\mathcal{L}} / 2}$ then completes the proof.

\section{Appendix C. A Version of the Feynman-Kac Formula for Perturbations of THE BochNeR LAPLACIAN}

This appendix is concerned with a proof of formula $(\sqrt{62})$. The strategy followed here is a combination of ideas as presented by Simon [63, Chapter V], Bismut [64, Chapitre IX], and Wittich [58]. The core portion of the proof is a version of Itô's formula for sections in line bundles, which will be derived first. The remaining part is an approximation argument.

We will use the same notation as in the main text, so $\mathcal{L}$ is a Hermitian line bundle with a connection $\nabla$ and an associated metric-preserving horizontal transport $H$. The $d$-dimensional base manifold $\mathcal{M}$ is complete with respect to the topology induced by a Riemannian metric. As usual, the Brownian motion in $\mathcal{M}$ with the diffusion constant $D>0$ and the starting point $x$ is denoted by $\mathrm{B}$, and the underlying probability measure by $\mathbb{P}_{x}^{D}$. Moreover, $\mathcal{M}$ is assumed to be Brownian-complete and its Ricci curvature bounded from below.

The Bochner Laplacian is denoted by $\Delta^{\mathcal{L}}$. An additive perturbation to $-D \Delta^{\mathcal{L}}$ by a function $q$ as discussed in Definition 27 results in the Schrödinger operator $S_{D, q}^{\mathcal{L}}$. At first, we focus on the unperturbed case.

Lemma C.1. Given a smooth section $\psi$ in $\mathcal{L}$, then for $t \geq 0$

$$
H_{\mathrm{B}, t}^{-1} \psi\left(\mathrm{B}_{t}\right)=\psi\left(\mathrm{B}_{0}\right)+\sum_{k=1}^{d} \int_{0}^{t} H_{\mathrm{B}, r}^{-1} \nabla_{E_{k}} \psi\left(\mathrm{B}_{r}\right)\left\langle E_{k}^{b}, \delta \mathrm{B}\right\rangle_{r}
$$

relates the inverse of the horizontal transport $H_{\mathrm{B}}$. along $\mathrm{B}$ and the connection $\nabla$ in a Stratonovich-integral equation. As usual, the right-hand side is invariant 
with respect to the particular choice of the section $\left\{E_{k}\right\}_{k=1}^{d}$ in a local orthonormal frame bundle. The brackets $\langle\cdot, \cdot\rangle$ denote a dual pairing, here with the one-form $E_{k}^{b}=g\left(E_{k}, \cdot\right)$.

Proof. By localization [51], it suffices to check this on a stochastic interval $\llbracket 0, \tau \rrbracket$, where $\tau$ is the exit time of $\mathrm{B}$ from the chart domain $U_{j}$ containing the starting point $\mathrm{B}_{0}$. The proof is accomplished using a local formulation of horizontal transport. To this end, we select a local trivialization $\xi_{j}$ and reference section $s_{j}$ around the $\mathrm{B}_{0}$ and associate with each section $\psi$ the representing function $\psi_{j}$ satisfying $\left.\psi\right|_{U_{j}}=\psi_{j} s_{j}$. The so-called connection one-form determined by $\nabla$ has a local representative $\alpha_{j}$ that satisfies $\nabla_{X}\left(\psi_{j} s_{j}\right)=\left(X\left(\psi_{j}\right)-i \alpha_{j}(X) \psi_{j}\right) s_{j}$ for all smooth $\psi$ and vector fields $X \in \Upsilon(\mathcal{M})$.

To simplify the notation, we define semimartingales $Y$ and $Z$ on $\llbracket 0, \tau \rrbracket$ by

$$
\mathrm{Y}_{t}:=\psi_{j}\left(\mathrm{~B}_{t}\right) \text { and } \mathrm{Z}_{t}:=e^{-i \int_{0}^{t}\left\langle\alpha_{j}, \delta \mathrm{B}\right\rangle},
$$

and use the shorthand $\delta \mathrm{W}^{(k)}=\left\langle E_{k}^{b}, \delta \mathrm{B}\right\rangle$, which represents the components of a Brownian motion $\mathrm{W}$ in $\mathbb{R}^{d}$ that is restricted to the stochastic interval. In conjunction with Stratonovich stochastic integrals, an integration by parts rule applies,

$$
\begin{aligned}
\mathrm{Z}_{t} \mathrm{Y}_{t}-\mathrm{Y}_{0} & =\int_{0}^{t} \mathrm{Z}_{r} \delta \mathrm{Y}_{r}+\int_{0}^{t} \mathrm{Y}_{r} \delta \mathrm{Z}_{r} \\
& =\sum_{k=1}^{d} \int_{0}^{t} \mathrm{Z}_{r} E_{k}\left(\psi_{j}\right)\left(\mathrm{B}_{r}\right) \delta \mathrm{W}_{r}^{(k)}-i \sum_{k=1}^{d} \int_{0}^{t} \mathrm{Z}_{r} \mathrm{Y}_{r} \alpha_{j}\left(E_{k}\right)\left(\mathrm{B}_{r}\right) \delta \mathrm{W}_{r}^{(k)} \\
& =\sum_{k=1}^{d} \int_{0}^{t} \mathrm{Z}_{r}\left(E_{k}\left(\psi_{j}\right)-i \alpha_{j}\left(E_{k}\right) \psi_{j}\right)\left(\mathrm{B}_{r}\right) \delta \mathrm{W}_{r}^{(k)},
\end{aligned}
$$

and after reinserting the definitions of $Y$ and $Z$, we obtain an identity which, together with the localized expression for reverse horizontal transport, shows that both sides of equation (84) are the same scalars multiplying $s_{j}\left(\mathrm{~B}_{0}\right)$.

Proposition C.2. With the same notation as in the preceding lemma, a version of the Itô formula in fiber bundles is expressed as

$$
H_{B, t}^{-1} \psi\left(\mathrm{B}_{t}\right)=\psi\left(\mathrm{B}_{0}\right)+\sum_{k=1}^{d} \int_{0}^{t} H_{\mathrm{B}, r}^{-1} \nabla_{E_{k}} \psi\left(\mathrm{B}_{r}\right) d \mathrm{~W}_{r}^{(k)}+\int_{0}^{t} H_{\mathrm{B}, r}^{-1} D \Delta^{\mathcal{L}} \psi\left(\mathrm{B}_{r}\right) d r .
$$

Proof. As the first step of the proof, we repeat the calculation in the preceding lemma, with $\mathrm{Y}_{t}$ replaced by $\mathrm{Y}_{t}^{(k)}:=\left(E_{k}\left(\psi_{j}\right)-i \alpha_{j}\left(E_{k}\right) \psi_{j}\right)\left(\mathrm{B}_{t}\right)$, which yields

$$
\begin{aligned}
\mathrm{Z}_{t} \mathrm{Y}_{t}^{(k)}-\mathrm{Y}_{0}^{(k)}=\sum_{l=1}^{d} & \int_{0}^{t} \mathrm{Z}_{r}\left(E_{l}\left(E_{k}\left(\psi_{j}\right)\right)-\left(\operatorname{Cov}_{l} E_{k}\right) \psi_{j}-i E_{l}\left(\alpha_{j}\left(E_{k}\right)\right)-\right. \\
& \left.i \alpha_{j}\left(\operatorname{Cov}_{l} E_{k}\right)-i \alpha_{j}\left(E_{l}\right)\left(E_{k}\left(\psi_{j}\right)-i \alpha_{j}\left(E_{k}\right) \psi_{j}\right)\right)\left(\mathrm{B}_{r}\right) \delta \mathrm{W}_{r}^{(l)} .
\end{aligned}
$$

The covariant derivative of the frame vectors enters because those are not horizontally transported along $\mathrm{B}$. 
Now we convert equations 88 and (90) to Itô differentials and insert the stochastic integral expression for $Z Y^{(k)}$ into the cross variation emerging from equation (88),

$$
\begin{aligned}
\mathrm{Z}_{t} \mathrm{Y}_{t}-\mathrm{Y}_{0}= & \sum_{k=1}^{d} \int_{0}^{t} \mathrm{Z}_{r} \mathrm{Y}_{r}^{(k)} \delta \mathrm{W}^{(k)}+\frac{1}{2} \sum_{k=1}^{d}\left[\mathrm{ZY}^{(k)}, \mathrm{W}^{(k)}\right]_{t} \\
= & \sum_{k=1}^{d} \int \mathrm{Z}_{r} \mathrm{Y}_{r}^{(k)} d \mathrm{~W}^{(k)}+\frac{1}{2} \sum_{k, l=1}^{d} \int_{0}^{t} \mathrm{Z}_{r}\left(\left(E_{l}\left(E_{k}\left(\psi_{j}\right)\right)-\left(\operatorname{Cov}_{l} E_{k}\right) \psi_{j}\right.\right. \\
& \left.\left.-i E_{l}\left(\alpha_{j}\left(E_{k}\right)\right)-i \alpha_{j}\left(\operatorname{Cov}_{l} E_{k}\right)\right)\left(\mathrm{B}_{r}\right)-i \alpha_{j}\left(E_{l}\right) \mathrm{Y}_{r}^{(k)}\right) d\left[\mathrm{~W}^{(l)}, \mathrm{W}^{(k)}\right]_{r} .
\end{aligned}
$$

After contracting the summation indices with the cross variation $\left[\mathrm{W}^{(l)}, \mathrm{W}^{(k)}\right]_{r}=$ $2 D \delta_{l k} r$, a similar identification as in the preceding lemma and the differential operator expression obtained for $\Delta^{\mathcal{L}}$ proves formula (89).

Consequence C.3. If $\psi \in L^{2}(h m)$ and $\mathbb{E}_{x}^{D}[\bullet]$ denotes the expectation with respect to the Brownian motion starting at $x \in \mathcal{M}$, then for $t \geq 0$ the semigroup generated by $D \Delta^{\mathcal{L}}$ can be represented as

$$
e^{t D \Delta^{\mathcal{L}}} \psi(x)=\mathbb{E}_{x}^{D}\left[H_{\mathrm{B}, t}^{-1} \psi\left(\mathrm{B}_{t}\right)\right]
$$

Proof. First, we assume $\psi \in C_{c \mathcal{L}}^{\infty}(\mathcal{M})$ and abbreviate $P_{D, t}^{\mathcal{L}} \psi(x):=\mathbb{E}_{x}\left(H_{\mathrm{B}, t}^{-1} \psi\left(\mathrm{B}_{t}\right)\right]$. Since $H_{\mathrm{B}, t}^{-1}$ preserves the Hermitian metric $h$, each $P_{D, t}^{\mathcal{L}}$ is seen to be a bounded operator. Moreover, by the time reversal invariance of Brownian motion it is self-adjoint. Finally, the family $\left\{P_{D, t}^{\mathcal{L}}\right\}_{t \geq 0}$ forms a semigroup due to the Markov property

$$
\begin{aligned}
P_{D, t+s}^{\mathcal{L}} \psi(x) & =\mathbb{E}_{x}^{D}\left[H_{\mathrm{B}, t+s}^{-1} \psi\left(\mathrm{B}_{t+s}\right)\right] \\
& =\mathbb{E}_{x}^{D}\left[H_{\mathrm{B}, t}^{-1} \mathbb{E}_{\mathrm{B}_{t}}^{D}\left[H_{\mathrm{B}, s}^{-1} \psi\left(\mathrm{B}_{s}\right)\right]\right]=P_{D, t}^{\mathcal{L}}\left(P_{D, s}^{\mathcal{L}} \psi\right)(x)
\end{aligned}
$$

valid for $s, t \geq 0$. To verify that both sides of (93) are identical, we note that the generators agree on $\psi \in C_{c \mathcal{L}}^{\infty}(\mathcal{M})$, because $P_{D, t}^{\mathcal{L}} \psi$ satisfies the same integral equation as $e^{t D \Delta^{\mathcal{L}}} \psi$

$$
\begin{aligned}
P_{D, t}^{\mathcal{L}} \psi(x) & =\mathbb{E}_{x}^{D}\left[H_{\mathrm{B}, t}^{-1} \psi\left(\mathrm{B}_{t}\right)\right] \\
& =\mathbb{E}_{x}^{D}\left[\psi\left(\mathrm{B}_{0}\right)+\int_{0}^{t} H_{\mathrm{B}, s}^{-1} D \Delta^{\mathcal{L}} \psi\left(\mathrm{B}_{s}\right) d s\right] \\
& =\psi(x)+\int_{0}^{t} P_{D, s}^{\mathcal{L}} D \Delta^{\mathcal{L}} \psi(x) d s .
\end{aligned}
$$

By the definition of $P_{D, t}^{\mathcal{L}}$, the semigroup can be defined on all $\psi \in L^{2}(h m)$. Therefore, its generator defines a self-adjoint extension of $\left.D \Delta^{\mathcal{L}}\right|_{C_{c \mathcal{L}}^{\infty}(\mathcal{M})}$, but this is necessarily $D \Delta^{\mathcal{L}}$, because the latter is essentially self-adjoint on $C_{c \mathcal{L}}^{\infty}(\mathcal{M})$.

Theorem C.4. If the assumptions listed at the beginning of this appendix are satisfied, $\psi \in L^{2}(h m)$, and $q \in \mathcal{K}_{ \pm}\left(\mathbb{P}^{D}\right)$, then the semigroup $e^{-t S_{D, q}^{\mathcal{L}}}$ generated by the Schrödinger operator $S_{D, q}^{\mathcal{L}}$ has the probabilistic representation

$$
e^{-t S_{D, q}^{\mathcal{L}}} \psi(x)=\mathbb{E}_{x}^{D}\left[e^{-\int_{0}^{t} q\left(\mathrm{~B}_{s}\right) d s} H_{\mathrm{B}, t}^{-1} \psi\left(\mathrm{B}_{t}\right)\right],
$$


valid for $m$-almost every $x \in \mathcal{M}$.

Proof. First, we suppose $q$ is continuous, $\psi$ is a smooth section, and both are bounded. Then, along the lines of (89) and with the integration by parts rule,

$$
\begin{array}{r}
e^{-\int_{0}^{t} q\left(\mathrm{~B}_{s}\right) d s} H_{\mathrm{B}, t}^{-1} \psi\left(\mathrm{B}_{t}\right)=\psi\left(\mathrm{B}_{0}\right) \\
+\sum_{k=1}^{d} \int_{0}^{t} e^{-\int_{0}^{r} q\left(\mathrm{~B}_{s}\right) d s} H_{\mathrm{B}, r}^{-1} \nabla_{E_{k}} \psi\left(\mathrm{B}_{r}\right) d \mathrm{~W}_{r}^{(k)} \\
+\int_{0}^{t} e^{-\int_{0}^{r} q\left(\mathrm{~B}_{s}\right) d s} H_{\mathrm{B}, r}^{-1}\left(D \Delta^{\mathcal{L}} \psi-q \psi\right)\left(\mathrm{B}_{r}\right) d r .
\end{array}
$$

Since $q$ is bounded, the modification of the heat semigroup defined by inserting (100) in the expectation value of (93) has as its generator a self-adjoint extension of $\left.\left(D \Delta^{\mathcal{L}}-q\right)\right|_{C_{c \mathcal{L}}^{\infty}(\mathcal{M})}$. Again, by essential self-adjointness, this is seen to be the difference $-S_{D, q}^{\mathcal{L}}=D \Delta^{\mathcal{L}}-q$.

In the last step, we approximate the general case $q \in \mathcal{K}_{ \pm}\left(\mathbb{P}^{D}\right)$ by truncation. We define the net $\left\{q_{k}^{(l)}\right\}$ of bounded functions

$$
x \mapsto q_{k}^{(l)}(x):=\min \{\max \{q(x),-k\}, l\}
$$

indexed by $k, l \in \mathbb{N}$. Truncating $q \in \mathcal{K}_{ \pm}\left(\mathbb{P}^{D}\right)$ in this manner gives

$$
e^{-t S_{D, q_{k}^{(l)}}^{\mathcal{L}}} \psi(x)=\mathbb{E}_{x}^{D}\left[e^{-\int_{0}^{t} q_{k}^{(l)}\left(\mathrm{B}_{s}\right) d s} H_{\mathrm{B}, t}^{-1} \psi\left(\mathrm{B}_{t}\right)\right]
$$

valid for $m$-almost every $x$ by the above argument. Now, by monotone form convergence we obtain strong convergence on the left when consecutively first $l \rightarrow \infty$ and then $k \rightarrow \infty$, whereas on the right dominated convergence applies to both limits, because

$$
\mathbb{E}_{x}^{D}\left[e^{\int_{0}^{t} q^{-}\left(\mathrm{B}_{s}\right) d s} h_{\mathrm{B}_{t}}\left(\psi\left(\mathrm{B}_{t}\right), \psi\left(\mathrm{B}_{t}\right)\right)\right]<\infty
$$

since the negative part $q^{-} \in \mathcal{K}\left(\mathbb{P}^{D}\right)$ and $x \mapsto h_{x}(\psi(x), \psi(x))$ are $m$-integrable.

\section{REFERENCES}

[1] F. A. Berezin, Covariant and contravariant operator symbols, Math. USSR Izvestija 6 (1972) 1117-1151, russ. orig.: Izv. Akad. Nauk SSSR Ser. Mat. 36 (1972), 1134-1167.

[2] F. A. Berezin, Quantization, Math. USSR Izvestija 8 (1974) 1109-1165, russ. orig.: Izv. Akad. Nauk SSSR, Ser. Mat. 38 (1974), 1116-1175.

[3] J. R. Klauder, Continuous-representation theory. I. Postulates of continuous-representation theory, J. Math. Phys. 4 (1963) 1055-1058.

[4] J. R. Klauder, Continuous-representation theory. II. Generalized relation between quantum and classical dynamics, J. Math. Phys. 4 (1963) 1058-1073.

[5] J. R. Klauder, Continuous-representation theory. III. On functional quantization of classical systems, J. Math. Phys. 5 (1964) 177-187.

[6] J. R. Klauder, J. McKenna, Continuous-representation theory. V. Construction of a class of scalar boson field continuous representations, J. Math. Phys. 6 (1965) 68-87.

[7] J. R. Klauder, J. McKenna, D. G. Currie, On "diagonal" coherent-state representations for quantum-mechanical density matrices, J. Math. Phys. 6 (1965) 734-739.

[8] J. McKenna, J. R. Klauder, Continuous-representation theory. IV. Structure of a class of function spaces arising from quantum mechanics, J. Math. Phys. 5 (1964) 878-896.

[9] M. Bordemann, E. Meinrenken, M. Schlichenmaier, Toeplitz quantization of Kähler manifolds and $g l(n), n \rightarrow \infty$ limits, Commun. Math. Phys. 165 (1994) 281-296.

[10] M. Cahen, S. Gutt, J. Rawnsley, Quantization of Kähler manifolds. I. Geometric interpretation of Berezin's quantization, J. Geom. Phys. 7 (1990) 45-62.

[11] M. Cahen, S. Gutt, J. Rawnsley, Quantization of Kähler manifolds. II, Trans. Amer. Math. Soc. 337 (1993) 73-98. 
[12] M. Cahen, S. Gutt, J. Rawnsley, Quantization of Kähler manifolds. III, Lett. Math. Phys. 30 (1994) 291-305.

[13] M. Cahen, S. Gutt, J. Rawnsley, Quantization of Kähler manifolds. IV, Lett. Math. Phys. 34 (1995) 159-168.

[14] B. Kostant, Quantization and unitary representations. I. Prequantization, in: Lectures in modern analysis and applications, III, Springer, Berlin, 1970, pp. 87-208. Lecture Notes in Math., Vol. 170.

[15] J. Śniatycki, Geometric quantization and quantum mechanics, Springer, New York, 1980.

[16] J.-M. Souriau, Quantification géométrique, Comm. Math. Phys. 1 (1966) 374-398.

[17] A. Perelomov, Generalized coherent states and their applications, Texts and Monographs in Physics, Springer, Berlin, 1986.

[18] M. Schlichenmaier, Berezin-Toeplitz quantization of compact Kähler manifolds, in: A. Strasburger, S. Ali, J.-P. Antoine, J.-P. Gazeau, A. Odzijewicz (Eds.), Quantization, Coherent States and Poisson Structures, Proceedings of the XIV'th Workshop on Geometric Methods in Physics, Białowieża, 1995, Polish Scientific Publisher PWN, 1998, pp. 101-115.

[19] I. Daubechies, J. R. Klauder, Constructing measures for path integrals, J. Math. Phys. 23 (1982) 1806-1822.

[20] I. Daubechies, J. R. Klauder, Quantum-mechanical path integrals with Wiener measure for all polynomial Hamiltonians. II, J. Math. Phys. 26 (1985) 2239-2256.

[21] I. Daubechies, J. R. Klauder, True measures for real time path integrals, in: M. L. Gutzwiller, A. Inomata, J. R. Klauder, L. Streit (Eds.), Path Integrals from meV to MeV, Bielefeld Encounters in Physics and Mathematics, World Scientific, Singapore, 1986, pp. 425-432.

[22] J. R. Klauder, I. Daubechies, Measures for path integrals, Phys. Rev. Lett. 48 (1982) 117-120.

[23] J. R. Klauder, I. Daubechies, Quantum mechanical path integrals with Wiener measures for all polynomial Hamiltonians, Phys. Rev. Lett. 52 (1984) 1161-1164.

[24] I. Daubechies, J. R. Klauder, T. Paul, Wiener measures for path integrals with affine kinematic variables, J. Math. Phys. 28 (1987) 85-102.

[25] R. Alicki, J. R. Klauder, Quantization of systems with a general phase space equipped with a Riemannian metric, J. Phys. A 29 (1996) 2475-2483.

[26] R. Alicki, J. R. Klauder, J. Lewandowski, Landau-level ground state and its relevance for a general quantization procedure, Phys. Rev. A 48 (1993) 2538-2548.

[27] J. R. Klauder, Quantization on non-homogeneous manifolds, Int. J. Theor. Phys. 33 (1994) $509-522$.

[28] J. R. Klauder, E. Onofri, Landau levels and geometric quantization, Int. J. Mod. Phys. 4 (1989) 3939-3949.

[29] L. Charles, Feynman path integral and Toeplitz quantization, Helv. Phys. Acta 72 (1999) 341-355.

[30] P. Griffiths, J. Harris, Principles of algebraic geometry, Wiley, New York, 1978.

[31] F. Zhang, Complex Differential Geometry, Studies in Advanced Mathematics, AMS and International Press, Providence (R. I.), 2000.

[32] B. Simon, Quantum mechanics for Hamiltonians defined as quadratic forms, Princeton University Press, Princeton, N. J., 1971, princeton Series in Physics.

[33] M. Reed, B. Simon, Methods of Modern Mathematical Physics, Vol. II, Fourier analysis, self-adjointness, Academic Press, New York, 1975.

[34] T. Kato, Quadratic forms in Hilbert spaces and asymptotic perturbation series, Department of Mathematics, University of California, Berkeley, Calif., 1955.

[35] P. D. Lax, A. N. Milgram, Parabolic equations, in: Contributions to the theory of partial differential equations, no. 33 in Ann. of Math. Stud., Princeton University Press, Princeton, N. J., 1954, pp. 167-190.

[36] J.-L. Lions, Équations différentielles opérationnelles et problèmes aux limites, Vol. 111 of Die Grundlehren der mathmematischen Wissenschaften, Springer, Berlin, 1961.

[37] E. Nelson, Interaction of nonrelativistic particles with a quantized scalar field, J. Math. Phys. 5 (1964) 1190-1197.

[38] M. Reed, B. Simon, Methods of modern mathematical physics, Vol. I, Functional analysis, Academic Press, New York, 1980.

[39] J. Weidmann, Linear Operators in Hilbert spaces, Vol. 68 of Graduate Texts in Mathematics, Springer, New York, 1980. 
[40] D. Cichoń, Notes on unbounded Toeplitz operators in Segal-Bargmann spaces, Ann. Polon. Math. 64 (1996) 227-235.

[41] J. Janas, J. Stochel, Unbounded Toeplitz operators in the Segal-Bargmann space, II, J. Funct. Anal. 126 (1994) 419-447.

[42] D. Borthwick, A. Uribe, Almost complex structures and geometric quantization, Math. Res. Lett. 3 (1996) 845-861.

[43] V. I. Arnold, Mathematical methods of classical mechanics, 2nd Edition, no. 60 in Graduate Texts in Mathematics, Springer, Berlin, 1989.

[44] R. E. Greene, H. Wu, $C^{\infty}$-approximations of convex, subharmonic, and plurisubharmonic functions, Ann. Scient. Éc. Norm. Sup. 12 (1979) 47-84.

[45] E. B. Davies, Heat kernels and spectral theory, Cambridge Tracts in Mathematics, Cambridge University Press, Cambridge, 1989.

[46] K.-T. Sturm, Heat kernel bounds on manifolds, Math. Ann. 292 (1992) 149-162.

[47] N. Berline, E. Getzler, M. Vergne, Heat kernels and Dirac operators, no. 298 in Grundlehren der mathematischen Wissenschaften, Springer, 1992.

[48] B. Simon, A canonical decomposition for quadratic forms with applications to monotone convergence, J. Funct. Anal. 28 (1978) 377-385.

[49] R. Z. Khaśminskii, On positive solutions of the equation $A u+V u=0$, Theoret. Probab. Appl. 4 (1959) 309-318.

[50] A.-S. Sznitman, Brownian Motion, Obstacles and Random Media, Springer Monographs in Mathematics, Springer, Berlin, 1998.

[51] L. Schwartz, Semi-martingales sur des variétés, et martingales conformes sur des variétés analytiques complexes, no. 780 in Lecture Notes in Mathematics, Springer, Berlin, 1980.

[52] I. Karatzas, S. Shreve, Brownian motion and Stochastic Calculus, 2nd Edition, no. 113 in Graduate Texts in Mathematics, Springer, New York, 1991

[53] K. R. Parthasarathy, Probability measures on metric spaces, Academic Press, New York, 1967.

[54] E. B. Davies, Gaussian upper bounds for the heat kernel of some second-order operators on Riemannian manifolds, J. Funct. Anal. 80 (1988) 16-32.

[55] B. Bodmann, H. Leschke, S. Warzel, A rigorous path integral for quantum spin using flatspace Wiener regularization, J. Math. Phys. 40 (1999) 2549-2559.

[56] E. Onofri, A note on coherent state representations of Lie groups, J. Math. Phys. 16 (1975) 1087-1089.

[57] B. Bodmann, H. Leschke, S. Warzel, A rigorous path-integral formula for quantum spin via planar Brownian motion, in: R. Casalbuoni, R. Giachetti, V. Tognetti, R. Vaia, P. Verrucchi (Eds.), Path Integrals from peV to TeV, World Scientific, Singapore, 1999, pp. 173-176.

[58] O. Wittich, A transformation of a Feynman-Kac formula for holomorphic families of type B, J. Math. Phys. (2000) 244-259.

[59] B. G. Bodmann, Relating resolvents of Berezin-Toeplitz operators by an invariance property of Brownian motion, in preparation.

[60] S. Bergman, The kernel function and conformal mapping, 2nd Edition, no. 5 in Amer. Math. Soc. Survey, AMS, Providence (R. I.), 1970.

[61] H. L. Cycon, R. G. Froese, W. Kirsch, B. Simon, Schrödinger operators, with application to quantum mechanics and global geometry, Texts and Monographs in Physics, Springer, Berlin, 1987.

[62] L. I. Hedberg, Approximation in Sobolev spaces and nonlinear potential theory, in: Nonlinear functional analysis and its applications, Part 1 (Berkeley, Calif., 1983), Amer. Math. Soc., Providence, RI, 1986, pp. 473-480.

[63] B. Simon, Functional integration and quantum physics, Academic Press, New York, 1979.

[64] J.-M. Bismut, Mécanique aléatoire, no. 866 in Lecture Notes in Mathematics, Springer, Berlin, 1981.

Bernhard G. Bodmann, 337 Jadwin Hall, Physics Department, Princeton University, PRINCETON, NJ 08544

E-mail address: bgb@princeton.edu 